Supporting Information

\title{
Structure Determination of Mycolactone C via Total Synthesis
}

Ted C. Judd ${ }^{\mathrm{a}}$, Alexander Bischoff ${ }^{\mathrm{a}}$, Yoshito Kishi ${ }^{\mathrm{a} *}$, Sarojini Adusumilli ${ }^{b}$, Pamela L. C. Small

${ }^{a}$ Department of Chemistry and Chemical Biology, Harvard University,

12 Oxford Street, Cambridge, Massachusetts 02138

${ }^{\mathrm{b}}$ Department of Microbiology, University of Tennessee,

Knoxville, Tennessee 37996 


\section{General Procedures and Methods}

Except as otherwise indicated, reactions were carried out under an nitrogen atmosphere in flame- or oven-dried glassware. Reagents and solvents are commercial grade and were used as supplied, with the following exceptions: THF was distilled from sodium/benzophenone; toluene, diethyl ether and dichloromethane were purified by passing through two alumina (A-2) columns using the J. C. Myer (Glass Contour) system; benzene was distilled from calcium hydride and stored over $3 \AA \mathrm{MS}$; and triethylamine was distilled from calcium hydride and stored over potassium hydroxide. Reactions were monitored by thin layer chromatography (TLC) with 0.25-mm E. Merck pre-coated silica gel plates. Silica gel 60 for flash chromatography (particle size 0.040$0.063 \mathrm{~mm}$ ) was supplied by E. Merck. Yields refer to chromatographically and spectroscopically pure compounds unless otherwise noted. ${ }^{1} \mathrm{H}$ and ${ }^{13} \mathrm{C}$ NMR spectra were recorded on a Varian Inova $600 \mathrm{MHz}$, Varian Inova $500 \mathrm{MHz}$ or Varian Mercury $400 \mathrm{MHz}$ spectrometer. Chemical shifts are reported as $\delta$ values (ppm) relative to internal chloroform $(\delta 7.26)$, benzene $(\delta 7.15)$, methanol $(\delta 3.34)$, or acetone $(\delta 2.04)$ for ${ }^{1} \mathrm{H}$ spectra and either chloroform $(\delta 77.0)$, benzene $(\delta 128.0)$, methanol $(\delta 49.0)$, or acetone $(\delta 29.8)$ for ${ }^{13} \mathrm{C}$ spectra. Optical rotations were measured on a Perkin-Elmer model 241 polarimeter. Low- and high-resolution mass spectra were measured at the Harvard University Chemistry and Chemical Biology Mass Spectrometry Facility.
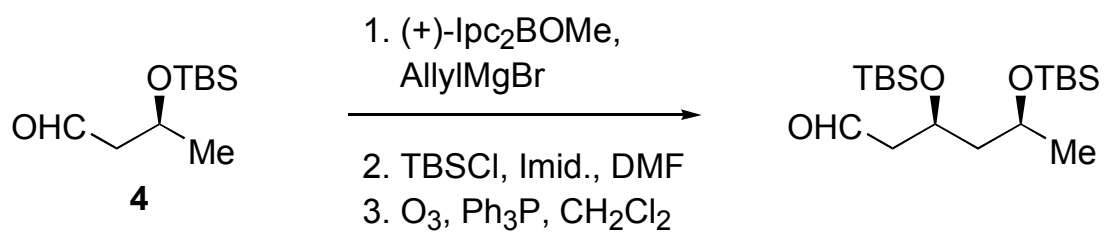

To a solution of $(+)-\mathrm{Ipc}_{2} \mathrm{BOMe}(516 \mathrm{mg}, 1.63 \mathrm{mmol})$ in $\mathrm{Et}_{2} \mathrm{O}(24 \mathrm{~mL})$ at $-78{ }^{\circ} \mathrm{C}$ was added allyl magnesium bromide $\left(1.63 \mathrm{~mL}, 1.63 \mathrm{mmol}, 1 \mathrm{M}\right.$ in $\left.\mathrm{Et}_{2} \mathrm{O}\right)$ by dropwise addition. After stirring $10 \mathrm{~min}$, the resulting solution was allowed to come to ambient temperature for $1 \mathrm{~h}$ and recooled to $-78^{\circ} \mathrm{C}$. The known aldehyde 4 (300 mg, $1.48 \mathrm{mmol}$; Paterson, I.; Craw, P. A. Tetrahedron Lett. 1989, 30, 5799) in $\mathrm{Et}_{2} \mathrm{O}$ was added in dropwise fashion and the reaction was allowed to stir for $1 \mathrm{~h}$ at $-78^{\circ} \mathrm{C}$. After warming to 0 ${ }^{\circ} \mathrm{C}$, the reaction was quenched by careful addition of aqueous $3 \mathrm{~N} \mathrm{NaOH}(1.1 \mathrm{~mL})$ and 
$30 \% \mathrm{H}_{2} \mathrm{O}_{2}(0.44 \mathrm{~mL})$ and stirred $12 \mathrm{hrs}$ at ambient temperature and diluted with $\mathrm{H}_{2} \mathrm{O}$. The layers were separated, and the aqueous layer was extracted $3 \times \mathrm{Et}_{2} \mathrm{O}$. The combined organic layers were washed $1 \times \mathrm{H}_{2} \mathrm{O}, 1 \times$ brine, and dried over $\mathrm{MgSO}_{4}$. Concentration in vacuo and purification by flash chromatography (9:1 Hex/EtOAc) afforded the corresponding allylic alcohol (243 $\mathrm{mg}, 67 \%$ yield) as a single diastereomer. (Note- in the case of the anti-1,3 diols used to prepare 11c and 11d, the diastereoselectivity was ca. 8:1 in favor of the desired product. The minor diastereomer was seperated following DiBAl$\mathrm{H}$ reduction at a later stage vide supra).

A solution of the allylic alcohol described above, TBSCl, and imidazole in DMF $(800 \mu \mathrm{L}$ ) was stirred for $2.5 \mathrm{~h}$ at ambient temperature. The reaction was quenched by dilution with $\mathrm{Et}_{2} \mathrm{O}$ and $\mathrm{H}_{2} \mathrm{O}$. The layers were separated, and the aqueous layer was extracted $2 \mathrm{X} \mathrm{Et}_{2} \mathrm{O}$. The combined organic layers were washed $2 \mathrm{x} \mathrm{H}_{2} \mathrm{O}, 1 \mathrm{x}$ brine, and dried over $\mathrm{MgSO}_{4}$. Concentration in vacuo and purification by flash chromatography (20:1 Hex/EtOAc) afforded the corresponding bis TBS-protected diol as a colorless oil (346 mg, 97\% yield). $[\alpha]^{23}{ }_{\mathrm{D}}=-2.4(\mathrm{c} 1.4, \mathrm{MeOH}){ }^{1} \mathrm{H}$ NMR $\left(400 \mathrm{MHz}, \mathrm{CDCl}_{3}\right) \delta 5.85$ $(\mathrm{m}, 1 \mathrm{H}), 5.05(\mathrm{~m}, 1 \mathrm{H}), 5.01(\mathrm{~m}, 1 \mathrm{H}), 3.90(\mathrm{~m}, 1 \mathrm{H}), 3.79(\mathrm{~m}, 1 \mathrm{H}), 2.26(\mathrm{~m}, 1 \mathrm{H}), 2.16(\mathrm{~m}$, 1H), 1.67 (ddd, $J=13.6,6.4,6.4 \mathrm{~Hz}, 1 \mathrm{H}), 1.51$ (ddd, $J=13.6,6.4,6.4 \mathrm{~Hz}, 1 \mathrm{H}), 1.13(\mathrm{~d}, J=$ $6.4 \mathrm{~Hz}, 3 \mathrm{H}), 0.88(\mathrm{~s}, 18 \mathrm{H}), 0.05(\mathrm{~s}, 6 \mathrm{H}), 0.04(\mathrm{~s}, 6 \mathrm{H}) ;{ }^{13} \mathrm{C} \mathrm{NMR}\left(\mathrm{CDCl}_{3}, 100 \mathrm{MHz}\right)$ б 135.1, 116.8, 69.3, 65.8, 47.1, 41.8, 25.9 (x3), 25.8 (x3), 23.9, -4.3, -4.3, -4.6, -4.8; HRMS (ES+) 359.2804, calcd 359.2801.

Anti-1,3 bis TBS-protected diol for the preparation of 11c and 11d:

${ }^{1} \mathrm{H}$ NMR $\left(400 \mathrm{MHz}, \mathrm{CDCl}_{3}\right) \delta 5.85(\mathrm{~m}, 1 \mathrm{H}), 5.05(\mathrm{~d}, J=3.6 \mathrm{~Hz}, 1 \mathrm{H}), 5.02(\mathrm{~s}, 1 \mathrm{H}), 3.89$ (m, 1H), $3.82(\mathrm{~m}, 1 \mathrm{H}), 2.22(\mathrm{~m}, 2 \mathrm{H}), 1.64(\mathrm{ddd}, J=7.2,14.0,5.2 \mathrm{~Hz}, 1 \mathrm{H}), 1.47$ (ddd, $J=6.8,14.0,5.2 \mathrm{~Hz}, 1 \mathrm{H}), 1.15(\mathrm{~d}, J=6.0 \mathrm{~Hz}, 3 \mathrm{H}), 0.88(\mathrm{~s}, 18 \mathrm{H}), 0.06(\mathrm{~s}, 12 \mathrm{H})$.

Ozone was passed through a solution of bis TBS-protected diol (346 mg, 0.965 mmol) described above in $\mathrm{CH}_{2} \mathrm{Cl}_{2}(50 \mathrm{~mL})$ at $-78{ }^{\circ} \mathrm{C}$ until a blue solution was observed. Oxygen followed by nitrogen gas was bubbled through the solution followed by addition of triphenylphosphine (313 mg, $1.29 \mathrm{mmol}$ ). After stirring at room temperature for $3 \mathrm{~h}$, the solution was concentrated in vacuo and purified by flash chromatography (20:1 Hex/EtOAc) to furnish the corresponding aldehyde ( $337 \mathrm{mg}$, 97\% yield). ${ }^{1} \mathrm{H}$ NMR (400 $\left.\mathrm{MHz}, \mathrm{CDCl}_{3}\right) \delta 9.79(\mathrm{dd}, J=2.0,3.2 \mathrm{~Hz}, 1 \mathrm{H}), 4.32(\mathrm{~m}, 1 \mathrm{H}), 3.86(\mathrm{~m}, 1 \mathrm{H}), 2.56(\mathrm{ddd}, J=$ 
4.4, 15.2, 1.6 Hz, 1H), 2.49 (ddd, $J=7.2,16.0,3.6 \mathrm{~Hz}, 1 \mathrm{H}), 1.78$ (ddd, $J=13.6,8.0,5.6$ $\mathrm{Hz}, 1 \mathrm{H}), 1.56$ (ddd, $J=13.6,8.0,4.8 \mathrm{~Hz}, 1 \mathrm{H}), 1.15$ (d, $J=6.0,3 \mathrm{H}), 0.87$ (s, 9H), 0.86 (s, 9H), 0.07 (s, 3H), $0.04(\mathrm{~s}, 6 \mathrm{H}), 0.03(\mathrm{~s}, 3 \mathrm{H})$.

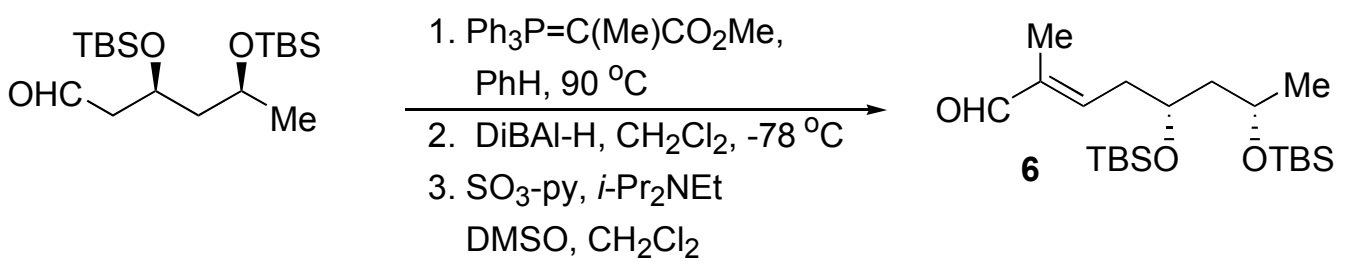

To a solution of the above aldehyde $(337 \mathrm{mg}, 0.934 \mathrm{mmol})$ in toluene $(10 \mathrm{~mL})$ was added carbethoxyethylidene triphenylphosphorane (792 mg, $2.19 \mathrm{mmol})$. The reaction was heated to $110{ }^{\circ} \mathrm{C}$ for $12 \mathrm{~h}$, cooled to room temperature, and then concentrated in vacuo. Flash chromatography $\left(20: 1 \mathrm{Hex} / \mathrm{E}_{2} \mathrm{O}\right)$ gave ester $5(357 \mathrm{mg}, 86 \%$ yield) as a colorless oil. $[\alpha]_{\mathrm{D}}^{23}=+9.4(\mathrm{c} 1.0, \mathrm{MeOH}){ }^{1} \mathrm{H}$ NMR $\left(400 \mathrm{MHz}, \mathrm{CDCl}_{3}\right) \delta 6.83$ (ddd, $J=$ 1.2, 7.6, $76 \mathrm{~Hz} 1 \mathrm{H}), 4.20$ (q, J=7.2 Hz, 2H), 3.89 (m, 2H), 2.35 (m, 1H), $2.30(\mathrm{~m}, 1 \mathrm{H})$, $1.82(\mathrm{~s}, 3 \mathrm{H}), 1.70(\mathrm{~m}, 1 \mathrm{H}), 1.49(\mathrm{~m}, 1 \mathrm{H}), 1.27$ (t, $J=7.2 \mathrm{~Hz}, 3 \mathrm{H}), 1.13(\mathrm{~d}, J=5.6 \mathrm{~Hz}, 3 \mathrm{H})$, $0.87(\mathrm{~s}, 18 \mathrm{H}), 0.04(\mathrm{~s}, 6 \mathrm{H}), 0.03(\mathrm{~s}, 6 \mathrm{H}) .{ }^{13} \mathrm{C} \mathrm{NMR}\left(100 \mathrm{MHz}, \mathrm{CDCl}_{3}\right) \delta$ 168.0, 138.7, $128.9,68.8,65.7,60.3,47.7,36.5,25.8$ (x 3), 25.8 (x 3), 23.9, 18.0, 17.9, 14.2, 12.6, -4.3, -4.6, -4.6, -4.8; HRMS (ES+) 445.3163, calcd 445.3169.

To a solution of ester $5(357 \mathrm{mg}, 0.803 \mathrm{mmol})$ in $\mathrm{CH}_{2} \mathrm{Cl}_{2}(10 \mathrm{~mL})$ at $-78{ }^{\circ} \mathrm{C}$ was slowly added DiBAl-H (2.0 ml, $2.0 \mathrm{mmol}, 1 \mathrm{M}$ in hexanes). The resulting solution was stirred at $-78{ }^{\circ} \mathrm{C}$ for $1.5 \mathrm{~h}$ and quenched with saturated aqueous potassium sodium tartrate $(25 \mathrm{~mL})$. The mixture was stirred vigorously for $6 \mathrm{~h}$ while warming to ambient temperature and diluted with EtOAc. The layers were separated, and the aqueous layer was extracted $2 \mathrm{X}$ EtOAc. The combined organic layers were washed $1 \mathrm{x} \mathrm{H}_{2} \mathrm{O}, 1 \mathrm{x}$ brine, and dried over $\mathrm{MgSO}_{4}$. Concentration in vacuo and purification by flash chromatography $(10: 1 \mathrm{Hex} / \mathrm{EtOAc})$ afforded the corresponding alcohol. $[\alpha]^{23}{ }_{\mathrm{D}}=-8.6(\mathrm{c} 1.2, \mathrm{MeOH}){ }^{1} \mathrm{H}$ NMR (400 MHz, $\left.\mathrm{CDCl}_{3}\right) \delta 5.43$ (ddd, $\left.J=1.2,7.2,7.2 \mathrm{~Hz} 1 \mathrm{H}\right), 3.99$ (s, 2H), 3.90 (m, 1H), $3.77(\mathrm{~m}, 1 \mathrm{H}), 2.19(\mathrm{~m}, 2 \mathrm{H}), 1.65(\mathrm{~s}, 3 \mathrm{H}), 1.63(\mathrm{~m}, 1 \mathrm{H}), 1.49(\mathrm{~m}, 1 \mathrm{H}), 1.12(\mathrm{~d}, J=6.4$ $\mathrm{Hz}, 3 \mathrm{H}), 0.86$ (s, $18 \mathrm{H}), 0.03$ (s, 3H), 0.03 (s, $3 \mathrm{H}), 0.02(\mathrm{~s}, 6 \mathrm{H}) ;{ }^{13} \mathrm{C} \mathrm{NMR}\left(\mathrm{CDCl}_{3}, 100\right.$ 
MHz,) $\delta 136.2,122.4,69.5,69.0,65.9,47.2,35.5,25.9$ (x 3), 25.8 (x3), 23.8, 18.1, 18.0, 13.9, -4.3 (x 2), -4.6, -4.8. HRMS (ES+) $403.3062(\mathrm{M}+\mathrm{H})$, calcd. 403.3064.

To a stirred solution of the above alcohol (45 mg, $112 \mu \mathrm{mol})$ in $\mathrm{CH}_{2} \mathrm{Cl}_{2}(1.2 \mathrm{ml})$ was added Dess-Martin periodane $(66 \mathrm{mg}, 156 \mu \mathrm{mol})$ in one portion. The resulting solution was stirred for $1 \mathrm{~h}$ and quenched by addition of saturated aqueous $\mathrm{NaHCO}_{3}$ and $\mathrm{Na}_{2} \mathrm{~S}_{2} \mathrm{O}_{4}$. Following dilution with $\mathrm{Et}_{2} \mathrm{O}$, the mixture was stirred vigorously for $20 \mathrm{~min}$. The layers were separated, and the aqueous layer was extracted $2 \times \mathrm{Et}_{2} \mathrm{O}$. The combined organic layers were washed 1 x 50\% saturated aqueous $\mathrm{NaHCO}_{3}, 1$ x $\mathrm{H}_{2} \mathrm{O}, 1$ x brine, and dried over $\mathrm{MgSO}_{4}$. Concentration in vacuo and purification by flash chromatography (20:1 Hex/Et $2 \mathrm{O}$ ) afforded aldehyde 6 (43 mg, 96\% yield) as a colorless oil. ${ }^{1} \mathrm{H}$ NMR (400 $\left.\mathrm{MHz}, \mathrm{CDCl}_{3}\right) \delta 9.41(\mathrm{~s}, 1 \mathrm{H}), 6.59(\mathrm{t}, J=7.6 \mathrm{~Hz}, 1 \mathrm{H}), 3.99(\mathrm{~m}, 1 \mathrm{H}), 3.88(\mathrm{~m}, 1 \mathrm{H}), 2.52$ $(\mathrm{m}, 2 \mathrm{H}), 1.74(\mathrm{~s}, 3 \mathrm{H}), 1.72(\mathrm{~m}, 1 \mathrm{H}), 1.50(\mathrm{ddd}, J=12.8,7.2,5.2 \mathrm{~Hz}, 1 \mathrm{H}), 1.15(\mathrm{~d}, J=6.4$ $\mathrm{Hz}, 3 \mathrm{H}), 0.88$ (s, 9H), 0.87 (s, 9H), 0.06 (s, 3H), 0.05 (s, 3H), 0.04 (s, 6H).

Each diastereomer of the polyunsaturated carboxylic acid was prepared analogous to our previously reported conditions, see: Song, F.; Fidanze, S.; Benowitz; Kishi, Y. Org. Lett. 2002, 4, 647.

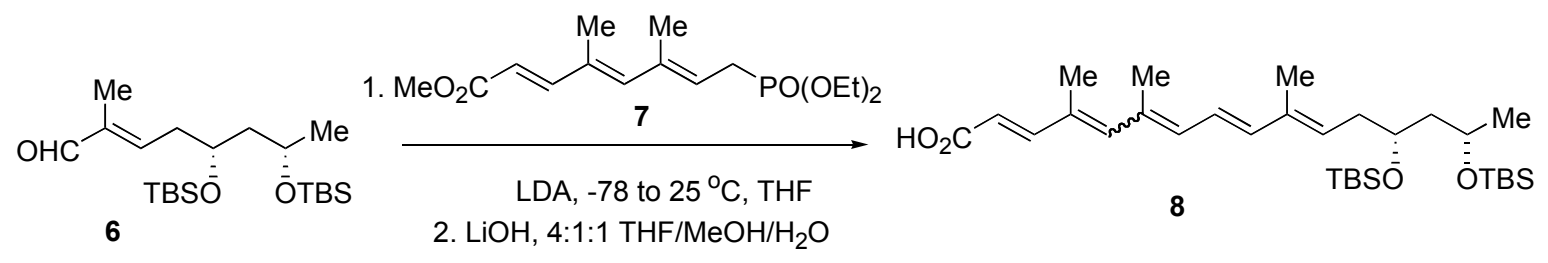

To a stirred solution of aldehyde 6 (32 mg, $79.0 \mu \mathrm{mol})$ and phosphonate 7 (50 mg, 158 umol; Gurjar, M. K.; Cherian, J; Heterocycles 2001, 55, 1095) in THF (0.6 mL) at $-78{ }^{\circ} \mathrm{C}$ was added dropwise freshly prepared LDA $(0.3 \mathrm{~mL}, 150 \mu \mathrm{mol}, 0.5 \mathrm{M}$ in THF). The resulting dark red solution was stirred at $-78{ }^{\circ} \mathrm{C}$ for $10 \mathrm{~min}$, then allowed to warm to $0{ }^{\circ} \mathrm{C}$ over $1 \mathrm{~h}$. After stirring for an additional $1 \mathrm{~h}$ at ambient temperature, the reaction was quenched by addition of saturated aqueous $\mathrm{NH}_{4} \mathrm{Cl}$ followed by dilution with EtOAc. The layers were separated, and the aqueous layer was extracted $3 \times$ EtOAc. The combined organic layers were washed $1 \times \mathrm{H}_{2} \mathrm{O}, 1 \mathrm{x}$ brine, and dried over $\mathrm{Na}_{2} \mathrm{SO}_{4}$. Concentration in 
vacuo and purification by flash chromatography (20:1 Hex/EtOAC) furnished the corresponding ester (50 $\mathrm{mg}, 96 \%)$ as a yellow oil. This ester exists as a 1:1 mixture of 4'Z:4'E isomers.

To a stirred solution of the above ester $(25 \mathrm{mg}, 44.4 \mu \mathrm{mol})$ in $4: 1: 1$ THF/MeOH/ $\mathrm{H}_{2} \mathrm{O}(1.4 \mathrm{~mL})$ was added $\mathrm{LiOH}\left(430 \mu \mathrm{l}, 0.43 \mathrm{mmol}, 1.0 \mathrm{M}\right.$ in $\left.\mathrm{H}_{2} \mathrm{O}\right)$. The resulting solution was protected from light and stirred for $18 \mathrm{~h}$ at ambient temperature. The reaction was quenched by addition of saturated aqueous $\mathrm{NH}_{4} \mathrm{Cl}$ followed by dilution with EtOAc. The layers were separated, and the aqueous layer was extracted $3 \times$ EtOAc. The combined organic layers were washed $1 \times \mathrm{H}_{2} \mathrm{O}, 1 \mathrm{x}$ brine, and dried over $\mathrm{Na}_{2} \mathrm{SO}_{4}$. Concentration in vacuo and purification by flash chromatography (4:1 Hex/EtOAC) furnished the acid $\mathbf{8}(23 \mathrm{mg}, 96 \%)$ as a yellow oil. This acid exists as a ca. 1:1 mixture of 4'Z:4'E isomers. $[\alpha]^{23}{ }_{\mathrm{D}}=+12.3(\mathrm{c} 0.76, \mathrm{MeOH}){ }^{1} \mathrm{H}$ NMR $\left(400 \mathrm{MHz}, \mathrm{CD}_{3} \mathrm{COCD}_{3}, 4^{\prime} \mathrm{Z}\right.$ isomer) $\delta 7.95(\mathrm{~d}, J=16.0 \mathrm{~Hz}, 1 \mathrm{H}), 6.54(\mathrm{dd}, J=14.8,10.8 \mathrm{~Hz}, 1 \mathrm{H}), 6.44(\mathrm{~s}, 1 \mathrm{H}), 6.37$ (d, $J=10.4 \mathrm{~Hz}, 1 \mathrm{H}), 6.17(\mathrm{~d}, \mathrm{~J}=11.2 \mathrm{~Hz}, 1 \mathrm{H}), 5.89$ (d, $J=15.6,1 \mathrm{H}), 5.71(\mathrm{dd}, J=8.0,16.0$ $\mathrm{Hz}, 1 \mathrm{H}), 3.93(\mathrm{~m}, 2 \mathrm{H}), 2.37(\mathrm{~m}, 2 \mathrm{H}), 2.01(\mathrm{~s}, 3 \mathrm{H}), 1.96(\mathrm{~s}, 3 \mathrm{H}), 1.84(\mathrm{~s}, 3 \mathrm{H}), 1.71(\mathrm{~m}$, 1H), $1.51(\mathrm{~m}, 1 \mathrm{H}), 1.14(\mathrm{~d}, J=6.0 \mathrm{~Hz}, 3 \mathrm{H}), 0.89$ (s, 9H), 0.08 (s, 6H). ${ }^{1} \mathrm{H}$ NMR (400 $\mathrm{MHz}, \mathrm{CD}_{3} \mathrm{COCD}_{3}, 4^{\prime} E$ isomer) $\delta 7.36(\mathrm{~d}, J=15.6 \mathrm{~Hz}, 1 \mathrm{H}), 6.56(\mathrm{dd}, J=14.8,10.8 \mathrm{~Hz}$, 1H), 6.48 (d, $J=14.8,1 \mathrm{H}), 6.44$ (s, 1H), 6.33 (d, $J=8.0 \mathrm{~Hz}, 1 \mathrm{H}), 5.87$ (d, $J=16.0 \mathrm{~Hz}, 1$ H), $5.71(\mathrm{dd}, J=8.0,16.0 \mathrm{~Hz}, 1 \mathrm{H}), 3.98(\mathrm{~m}, 2 \mathrm{H}), 2.07(\mathrm{~s}, 3 \mathrm{H}), 2.05(\mathrm{~s}, 3 \mathrm{H}), 1.84(\mathrm{~s}, 3 \mathrm{H})$, $1.68(\mathrm{~m}, 1 \mathrm{H}), 1.54(\mathrm{~m}, 1 \mathrm{H}), 1.15(\mathrm{~d}, J=5.6 \mathrm{~Hz}, 3 \mathrm{H}), 0.89(\mathrm{~s}, 9 \mathrm{H}), 0.08(\mathrm{~s}, 6 \mathrm{H}) .{ }^{13} \mathrm{C}$ NMR $\left(100 \mathrm{MHz}, \mathrm{CD}_{3} \mathrm{COCD}_{3}, 4^{\prime} Z\right.$ and $4^{\prime} E$ isomers); MS (ES+) $549.3800(\mathrm{M}+\mathrm{H})$, calcd. 549.3795 .

Anti-1,3 bis TBS-protected diol carboxylic acid for the preparation of $11 \mathbf{c}$ and 11d: ) ${ }^{1} \mathrm{H}$ NMR (400 MHz, $\mathrm{CD}_{3} \mathrm{COCD}_{3}, 4 \mathrm{Z}$ isomer) $\delta 7.95(\mathrm{~d}, J=15.6 \mathrm{~Hz}, 1 \mathrm{H}), 6.54$ (dd, $J=14.8,10.8 \mathrm{~Hz}, 1 \mathrm{H}), 6.44$ (d, $J=15.2 \mathrm{~Hz}, 1 \mathrm{H}), 6.39$ (d, $J=11.6 \mathrm{~Hz}, 1 \mathrm{H}), 6.19$ (d, $\mathrm{J}=10.4 \mathrm{~Hz}, 1 \mathrm{H}), 5.94(\mathrm{~d}, J=16.0,1 \mathrm{H}), 5.70(\mathrm{dd}, J=8.0,16.0 \mathrm{~Hz}, 1 \mathrm{H}), 3.99(\mathrm{~m}, 2 \mathrm{H})$, $2.39(\mathrm{~m}, 2 \mathrm{H}), 2.01(\mathrm{~s}, 3 \mathrm{H}), 1.96(\mathrm{~s}, 3 \mathrm{H}), 1.84(\mathrm{~s}, 3 \mathrm{H}), 1.62(\mathrm{~m}, 1 \mathrm{H}), 1.52(\mathrm{~m}, 1 \mathrm{H}), 1.14(\mathrm{~d}$, $J=6.0 \mathrm{~Hz}, 3 \mathrm{H}), 0.89$ (s, 9H), 0.08 (s, 6H). ${ }^{1} \mathrm{H}$ NMR $\left(400 \mathrm{MHz}, \mathrm{CD}_{3} \mathrm{COCD}_{3}, 4^{\prime} E\right.$ isomer $)$ $\delta 7.36(\mathrm{~d}, J=15.6 \mathrm{~Hz}, 1 \mathrm{H}), 6.56$ (dd, $J=14.8,10.8 \mathrm{~Hz}, 1 \mathrm{H}), 6.48$ (d, $J=14.4 \mathrm{~Hz}, 1 \mathrm{H})$, $6.46(\mathrm{~s}, 1 \mathrm{H}), 6.34(\mathrm{~s}, 1 \mathrm{H}), 5.88(\mathrm{~d}, J=15.6 \mathrm{~Hz}, 1 \mathrm{H}), 5.70(\mathrm{dd}, J=8.0,16.0 \mathrm{~Hz}, 1 \mathrm{H}), 3.98$ 
(m, 2H), $2.40(\mathrm{~m}, 2 \mathrm{H}), 2.07(\mathrm{~s}, 3 \mathrm{H}), 2.05(\mathrm{~s}, 3 \mathrm{H}), 1.84(\mathrm{~s}, 3 \mathrm{H}), 1.62(\mathrm{~m}, 1 \mathrm{H}), 1.52(\mathrm{~m}$, $1 \mathrm{H}), 1.15(\mathrm{~d}, J=6.0 \mathrm{~Hz}, 3 \mathrm{H}), 0.89(\mathrm{~s}, 9 \mathrm{H}), 0.08(\mathrm{~s}, 6 \mathrm{H})$.

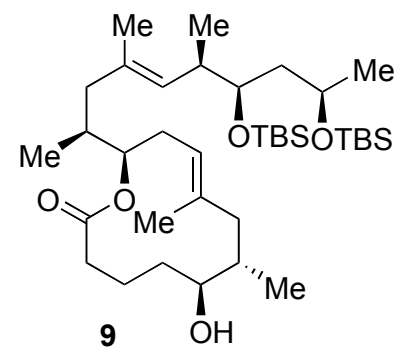

$+$

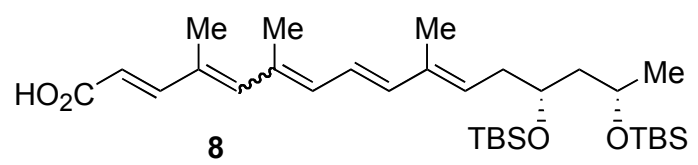

1. $\mathrm{Cl}_{3} \mathrm{C}_{6} \mathrm{CH}_{2} \mathrm{COCl}$, $i-\mathrm{Pr}_{2} \mathrm{NEt}$, DMAP, $\mathrm{PhH}$ 2. TBAF, THF, $25^{\circ} \mathrm{C}$

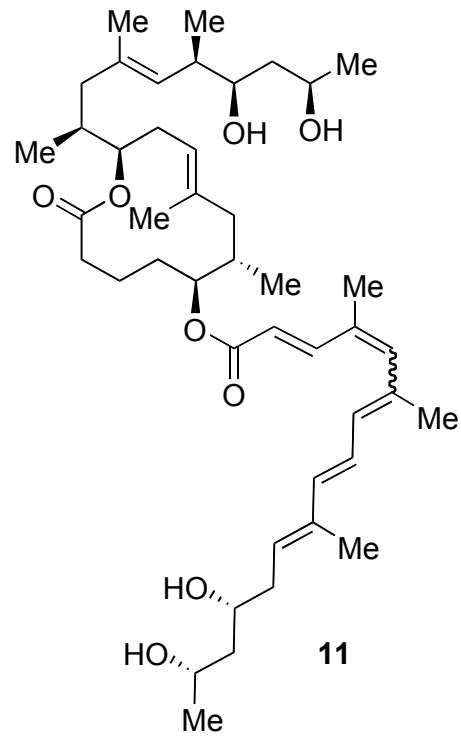

To a stirred solution of acid $8(19 \mathrm{mg}, 34.6 \mu \mathrm{mol})$ in benzene $(1.4 \mathrm{~mL})$ was added $i$ - $\operatorname{Pr}_{2} \mathrm{NEt}(16 \mu \mathrm{L}, 92.4 \mu \mathrm{mol}) \mathrm{Cl}_{3} \mathrm{C}_{6} \mathrm{H}_{2} \mathrm{COCl}(6.8 \mu \mathrm{L}, 43.8 \mu \mathrm{mol})$ and DMAP (11.3 mg, $92.4 \mu \mathrm{mol})$. After $20 \mathrm{~min}$, alcohol $9(15 \mathrm{mg}, 23.1 \mu \mathrm{mol})$ was added as a solution in benzene $(1.6 \mathrm{~mL})$. The reaction was protected from light and stirred $24 \mathrm{~h}$. The reaction was quenched by addition of saturated aqueous $\mathrm{NaHCO}_{3}$ and diluted with EtOAC. The layers were separated, and the aqueous layer was extracted 3 x EtOAc. The combined organic layers were washed $1 \times \mathrm{H}_{2} \mathrm{O}, 1 \times$ brine, and dried over $\mathrm{Na}_{2} \mathrm{SO}_{4}$. Concentration in vacuo and purification by flash chromatography (20:1 Hex/EtOAC) gave the corresponding ester ( $25 \mathrm{mg}, 91 \%$ yield) as a yellow oil. This ester exists as a ca. 1:1 mixture of 4 'Z:4'E isomers.

To a stirred solution of the above ester $10(25 \mathrm{mg}, 21.1 \mu \mathrm{mol})$ in THF $(2.3 \mathrm{~mL})$ was added TBAF $(190 \mu \mathrm{L}, 190 \mu \mathrm{mol}, 1 \mathrm{M}$ in THF). The reaction was protected from light and stirred $18 \mathrm{~h}$. The reaction was quenched by addition of saturated aqueous $\mathrm{NH}_{4} \mathrm{Cl}$ and diluted with EtOAC. The layers were separated, and the aqueous layer was extracted $3 \mathrm{x}$ EtOAc. The combined organic layers were washed $1 \times \mathrm{H}_{2} \mathrm{O}, 1 \mathrm{x}$ brine, and dried over 
$\mathrm{Na}_{2} \mathrm{SO}_{4}$. Concentration in vacuo and purification by flash chromatography (100:1 EtOAc/MeOH) gave the corresponding synthetic mycolactone $\mathbf{1 1}$ as a yellow oil (12.8 $\mathrm{mg}, 84 \%$ yield). The synthetic mycolactones exist as a ca. $1: 1$ mixture of $4{ }^{\prime} \mathrm{Z}: 4^{\prime} \mathrm{E}$ isomers. $[\alpha]^{23}=-15.5(\mathrm{c} 0.48, \mathrm{MeOH}){ }^{1} \mathrm{H}$ NMR $\left(500 \mathrm{MHz}, \mathrm{CD}_{3} \mathrm{COCD}_{3}, 4^{\prime} \mathrm{Z}\right.$ isomer $)$ $\delta 7.93(\mathrm{~d}, J=16.0 \mathrm{~Hz}, 1 \mathrm{H}), 6.51$ (dd, $J=11.0,15.5 \mathrm{~Hz}, 1 \mathrm{H}), 6.45$ (d, $J=15.5 \mathrm{~Hz}, 1 \mathrm{H})$, $6.32(\mathrm{~s}, 1 \mathrm{H}), 6.15$ (d, $J=11.0 \mathrm{~Hz}, 1 \mathrm{H}), 5.93(\mathrm{~d}, J=16.0 \mathrm{~Hz}, 1 \mathrm{H}), 5.72$ (dd, $J=7.5,15.5$ $\mathrm{Hz}, 1 \mathrm{H}), 5.12(\mathrm{~m}, 1 \mathrm{H}), 5.04(\mathrm{~d}, J=10.0 \mathrm{~Hz}, 1 \mathrm{H}), 4.88(\mathrm{~m}, 1 \mathrm{H}), 4.70(\mathrm{~m}, 1 \mathrm{H}), 4.23(\mathrm{dd}, J=$ 3.5, $3.5 \mathrm{~Hz}, 1 \mathrm{H}), 4.19$ (d, $J=3.5 \mathrm{~Hz}, 1 \mathrm{H}), 3.50(\mathrm{~m}, 1 \mathrm{H}), 2.49(\mathrm{~m}, 1 \mathrm{H}), 2.38(\mathrm{~m}, 2 \mathrm{H}), 2.34$ (m, 2H), 2.07-1.85 (m, 8H), $2.01(\mathrm{~s}, 3 \mathrm{H}), 1.99(\mathrm{~s}, 3 \mathrm{H}), 1.84(\mathrm{~s}, 3 \mathrm{H}), 1.69(\mathrm{~s}, 3 \mathrm{H}), 1.64(\mathrm{~s}$, $3 \mathrm{H}), 1.83(\mathrm{~m}, 1 \mathrm{H}), 1.70(\mathrm{~m}, 1 \mathrm{H}), 1.66(\mathrm{~m}, 1 \mathrm{H}), 1.55-1.60(\mathrm{~m}, 4 \mathrm{H}), 1.48(\mathrm{~m}, 1 \mathrm{H}), 1.38$ (m, 1H), 1.13 (d, $J=5.5 \mathrm{~Hz}, 3 \mathrm{H}), 1.11(\mathrm{~d}, J=6.0 \mathrm{~Hz}, 3 \mathrm{H}), 0.97$ (d, $J=7.0 \mathrm{~Hz}, 3 \mathrm{H}), 0.90$ (d, $J=6.5 \mathrm{~Hz}, 1 \mathrm{H}), 0.88(\mathrm{~d}, J=7.0 \mathrm{~Hz}, 3 \mathrm{H})$.

${ }^{1} \mathrm{H}$ NMR $\left(500 \mathrm{MHz}, \mathrm{CD}_{3} \mathrm{COCD}_{3}, 4^{\prime} E\right.$ isomer) $\delta 7.37$ (d, $\left.J=15.5 \mathrm{~Hz}, 1 \mathrm{H}\right), 6.52$ (dd, $J=$ 10.5, 15.0 Hz), 6.45 (s, $1 \mathrm{H}), 6.42(\mathrm{~d}, J=15.5 \mathrm{~Hz}, 1 \mathrm{H}), 6.34(\mathrm{~d}, J=12.0 \mathrm{~Hz}, 1 \mathrm{H}), 5.88(\mathrm{~d}$, $J=16.0 \mathrm{~Hz}, 1 \mathrm{H}), 5.72(\mathrm{dd}, J=7.5,15.5 \mathrm{~Hz}, 1 \mathrm{H}), 5.12(\mathrm{~m}, 1 \mathrm{H}), 5.04(\mathrm{~d}, J=10.0 \mathrm{~Hz}, 1 \mathrm{H})$, $4.88(\mathrm{~m}, 1 \mathrm{H}), 4.70(\mathrm{~m}, 1 \mathrm{H}), 4.22(\mathrm{~d}, J=3.5 \mathrm{~Hz}, 1 \mathrm{H}), 4.09(\mathrm{dd}, J=3.0,5.5 \mathrm{~Hz}, 1 \mathrm{H}), 3.50$ (m, 1H), $2.49(\mathrm{~m}, 1 \mathrm{H}), 2.38(\mathrm{~m}, 2 \mathrm{H}), 2.34(\mathrm{~m}, 2 \mathrm{H}), 2.07-1.85(\mathrm{~m}, 8 \mathrm{H}), 2.07(\mathrm{~s}, 3 \mathrm{H}), 1.96$ (s, 3H), $1.83(\mathrm{~s}, 3 \mathrm{H}), 1.71(\mathrm{~s}, 3 \mathrm{H}), 1.64(\mathrm{~s}, 3 \mathrm{H}), 1.83(\mathrm{~m}, 1 \mathrm{H}), 1.70(\mathrm{~m}, 1 \mathrm{H}), 1.66(\mathrm{~m}, 1 \mathrm{H})$, $1.55-1.60(\mathrm{~m}, 4 \mathrm{H}), 1.48(\mathrm{~m}, 1 \mathrm{H}), 1.38(\mathrm{~m}, 1 \mathrm{H}), 1.13(\mathrm{~d}, J=5.5 \mathrm{~Hz}, 3 \mathrm{H}), 1.11(\mathrm{~d}, J=6.0$ $\mathrm{Hz}, 3 \mathrm{H}), 0.97$ (d, $J=7.0 \mathrm{~Hz}, 3 \mathrm{H}), 0.90(\mathrm{~d}, J=6.5 \mathrm{~Hz}, 1 \mathrm{H}), 0.87(\mathrm{~d}, J=6.5 \mathrm{~Hz}, 3 \mathrm{H}) .{ }^{13} \mathrm{C}$ NMR (100 MHz, $\mathrm{CD}_{3} \mathrm{COCD}_{3}, 4^{\prime} E$ and $4^{\prime} Z$ isomer) $\delta 173.2$ (x 2), 166.9 (x 2), 151.2, 144.5, 143.2, 141.9, 141.0, 140.6, 137.3 (x 2), 136.6, 136.4 (x 2), 135.3, 134.4, 133.9, 133.4 (x 2), 132.1, 131.9, 131.8, 131.2 (x 3), 123.8 (x 4), 123.4 (x 2), 119.4, 117.1, 79.2 (x 3), 76.9 (x 2), 76.2, 76.2, 72.2 (x 3), 69.0 (x 2), 68.2 (x 2), 46.4 (x 2), 45.8 (x 2), 44.3 (x 2), 43.8, 40.5 (x 2), 38.0, 36.0 (x 2), 35.9, 35.3 (x 2), 32.8, 32.7, 31.5, 31.3, 24.6 (x 2), 24.4 (x 2), 20.9, 20.7, 20.6 (x 2), 20.5, 17.5, 17.1 (x 2), 17.0, 16.2, 15.9, 14.9 (x 2), 14.3(x 2), 12.7 (x 2). HRMS (ES+) $727.5143(\mathrm{M}+\mathrm{H})$, calcd 727.5149. UV (MeOH) $\lambda_{\max } 365 \mathrm{~nm}$ $(\log \varepsilon 4.69)$.

are included.

${ }^{1} \mathrm{H}$ NMR spectrum for each of the four diastereomers 11a, 11b, 11c, and 11d, 


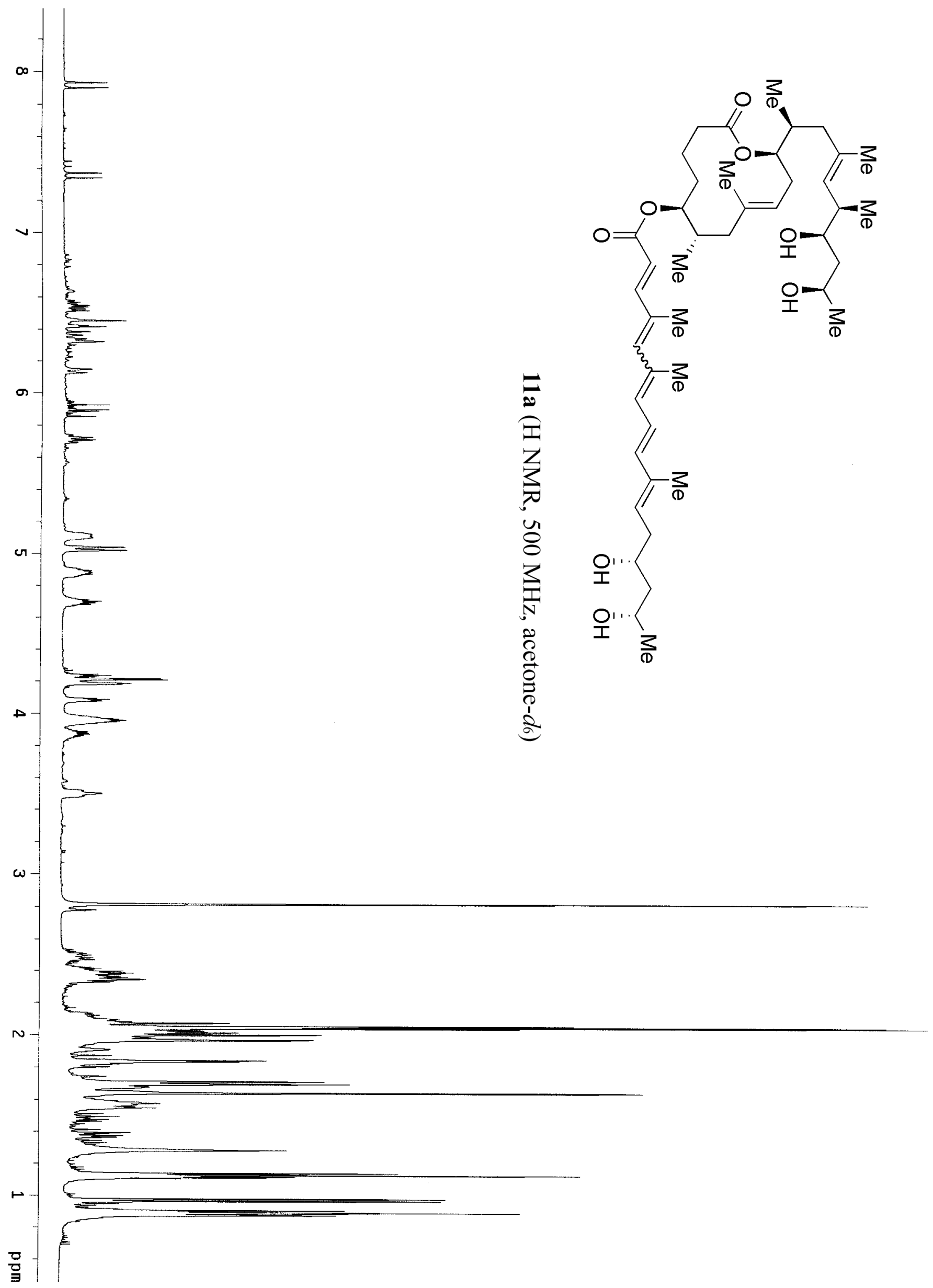




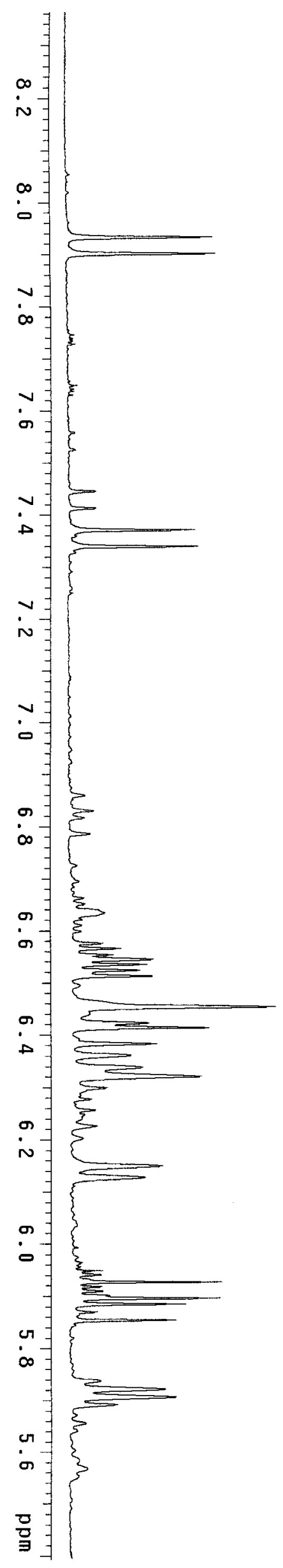

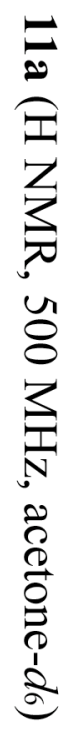




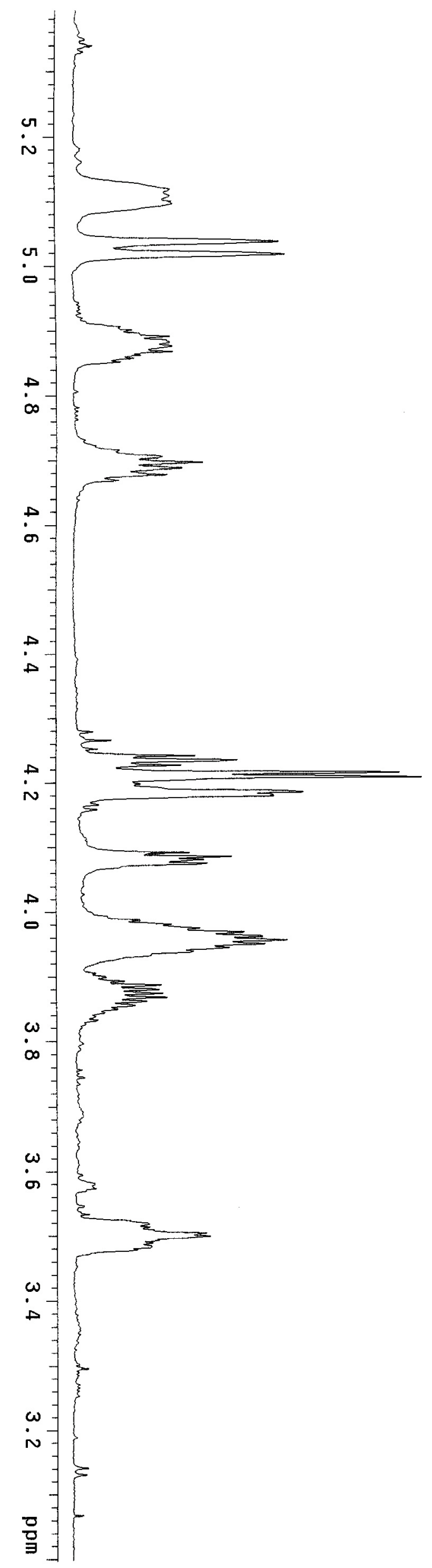

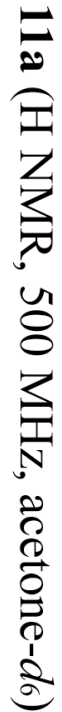




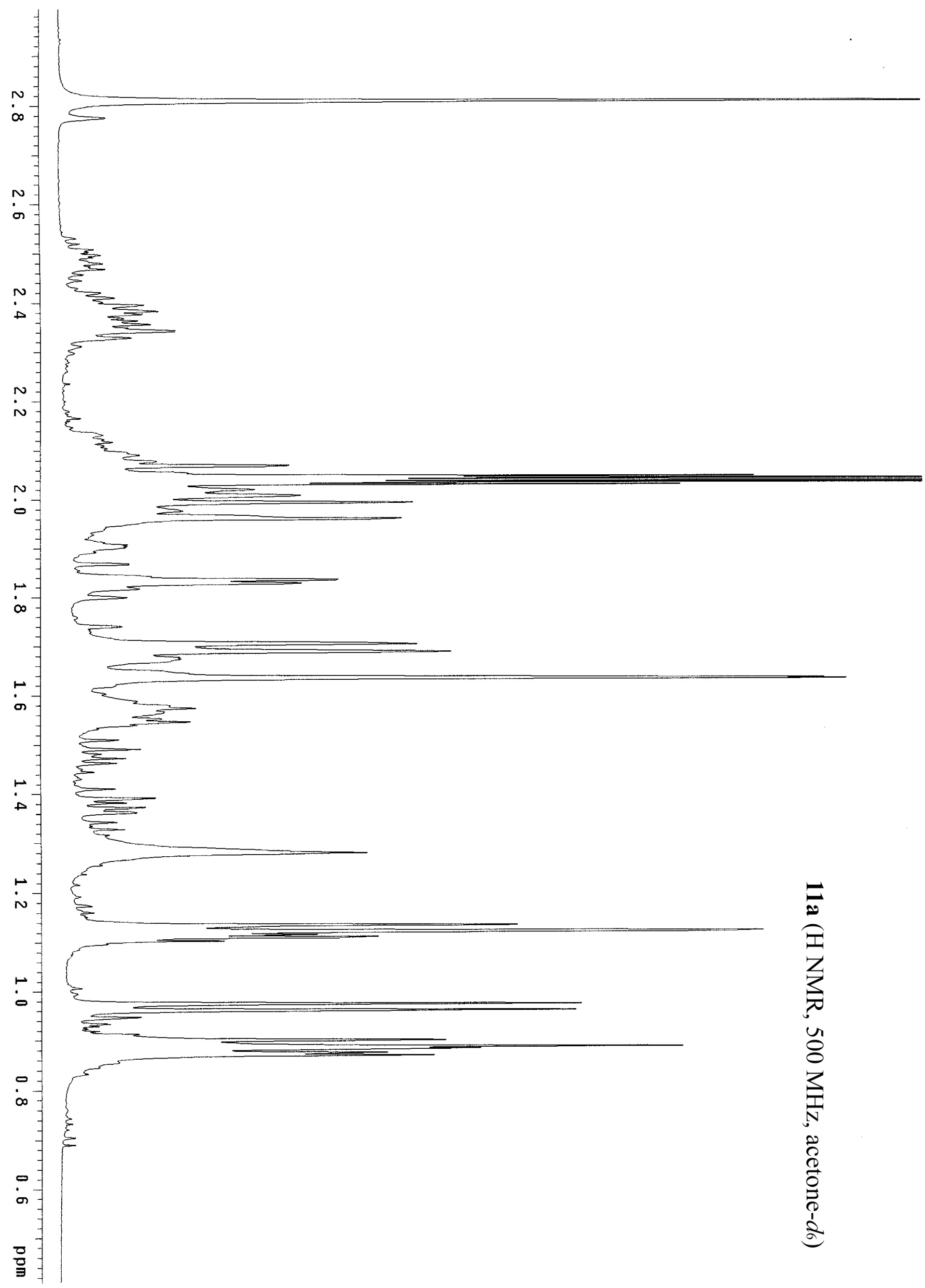




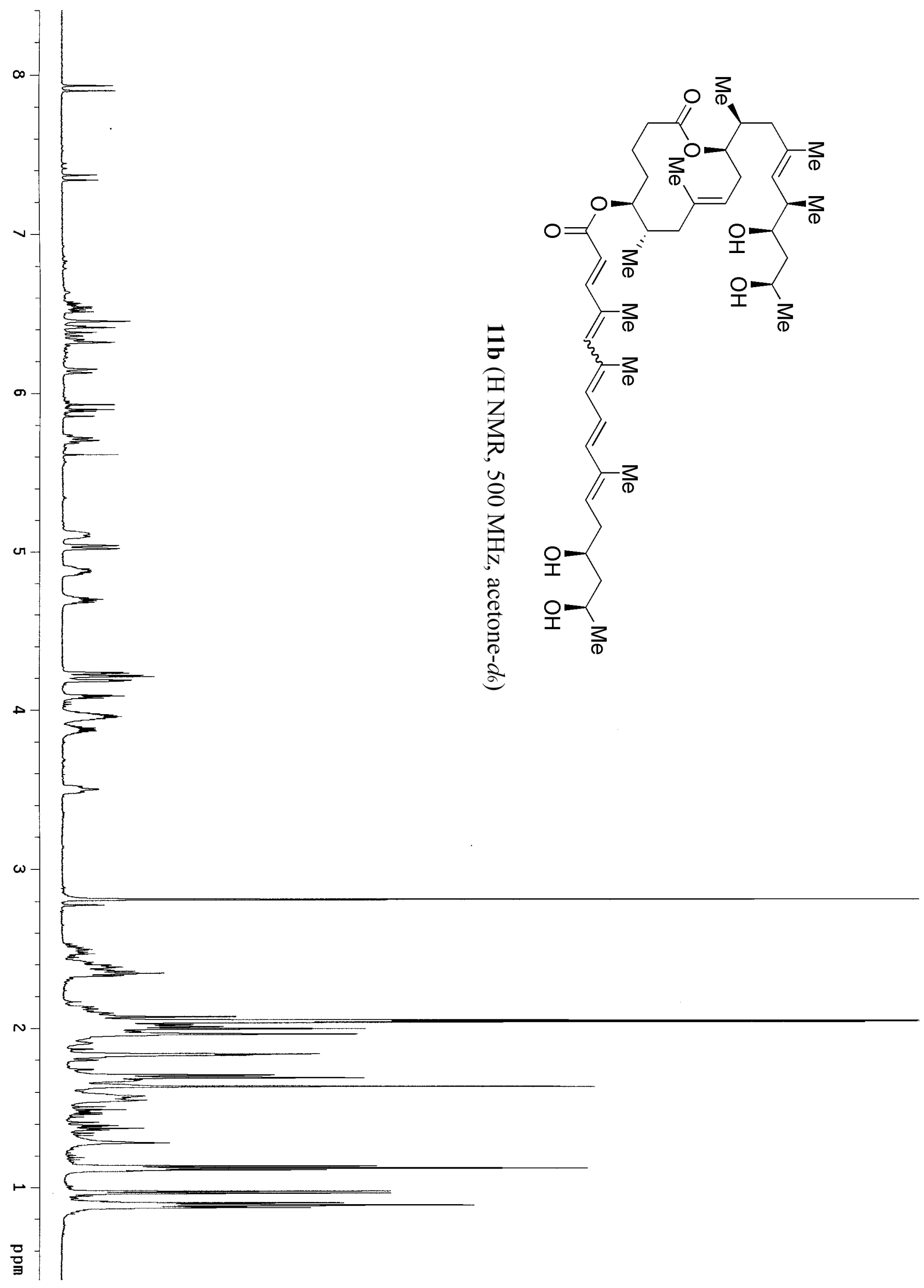




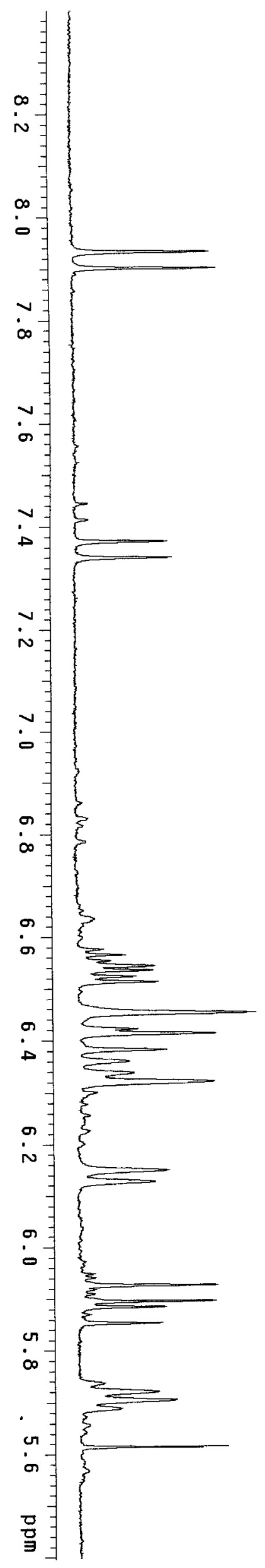

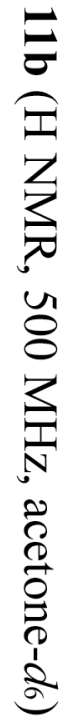




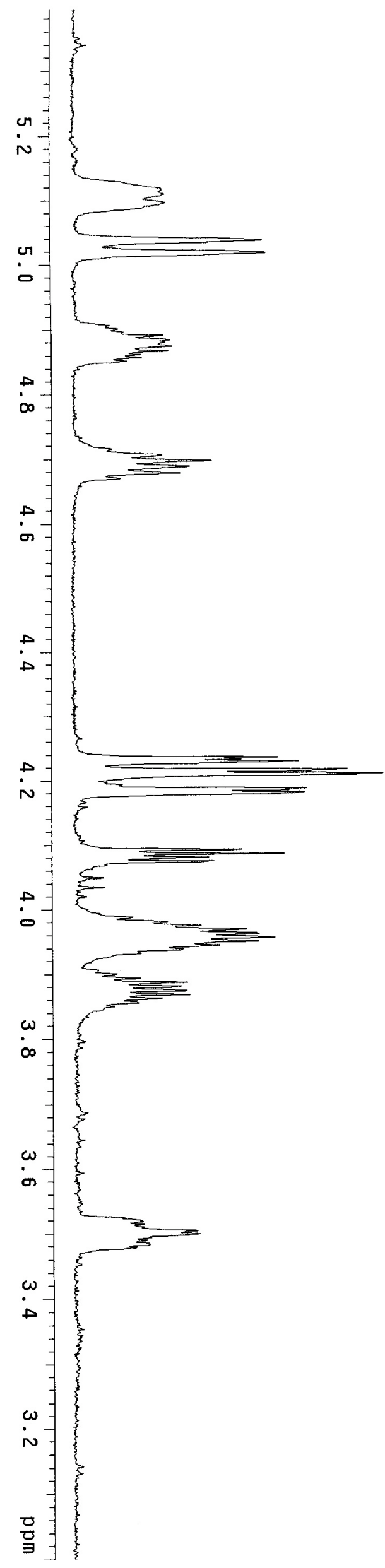

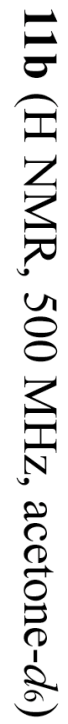




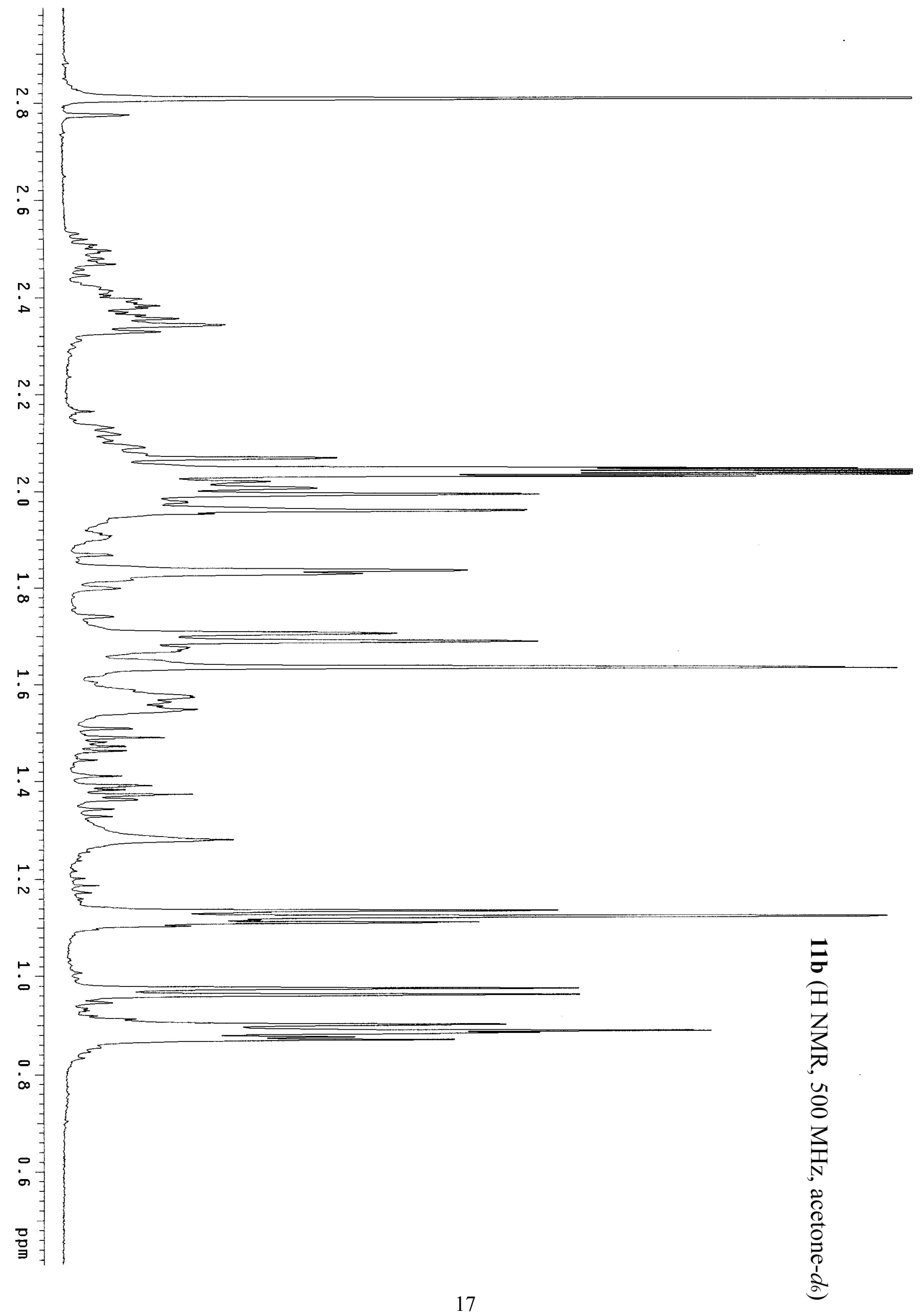




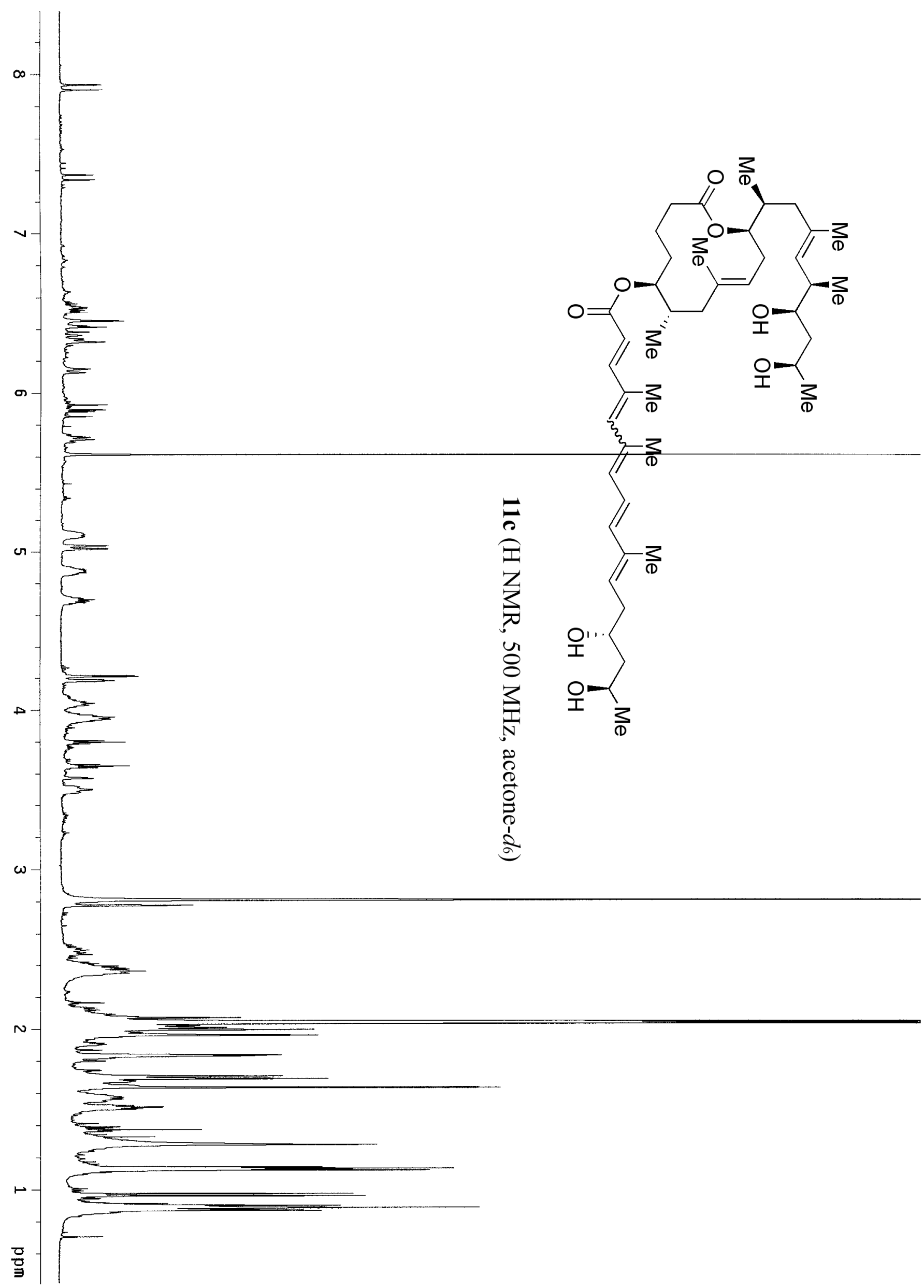




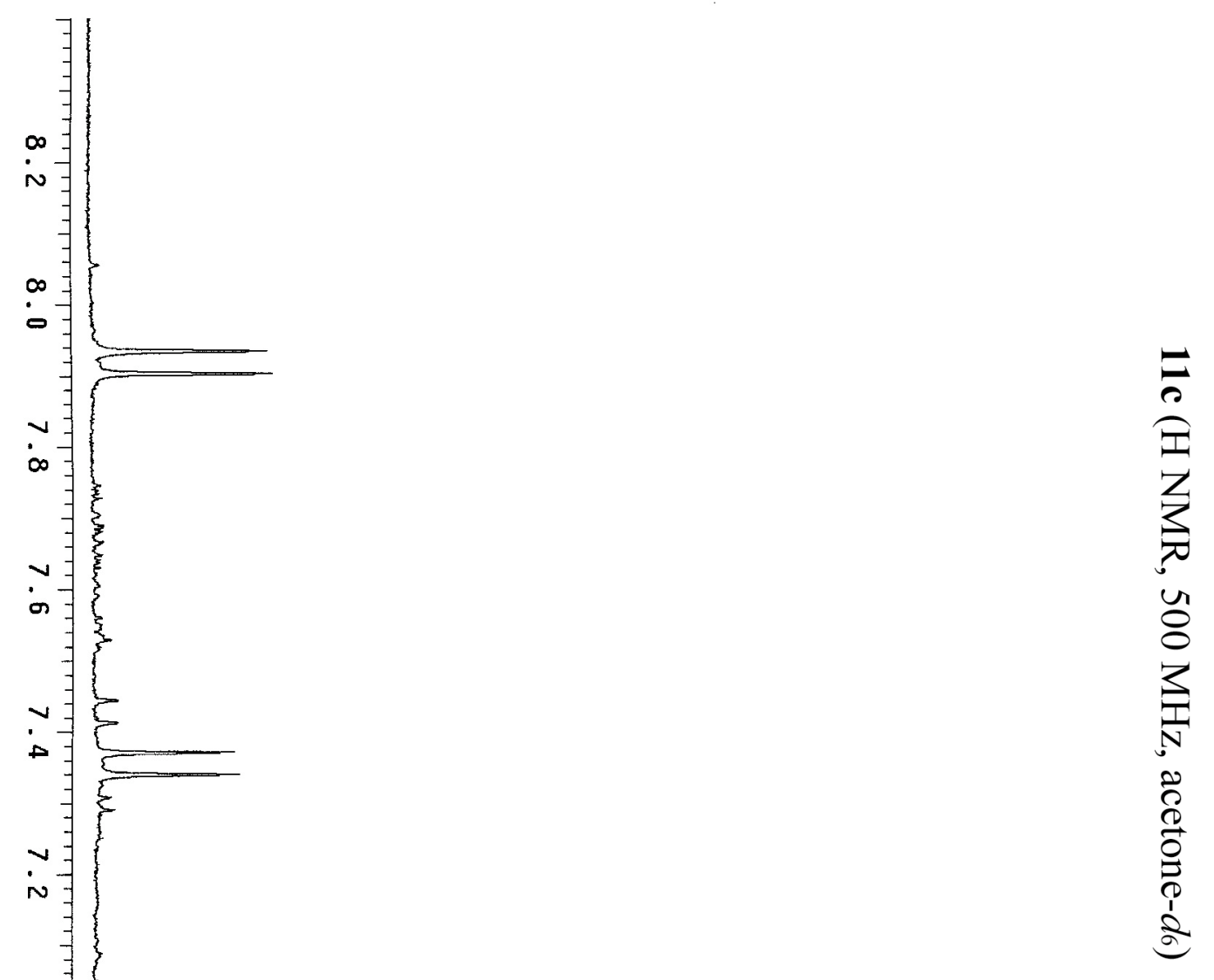

i

क

$\infty=$

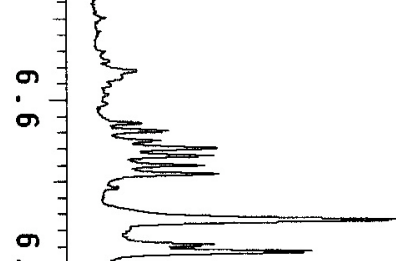

$\therefore 7=$

作主

-

:日

主

$\because$,

$\infty 1$

c)

in

을 青 

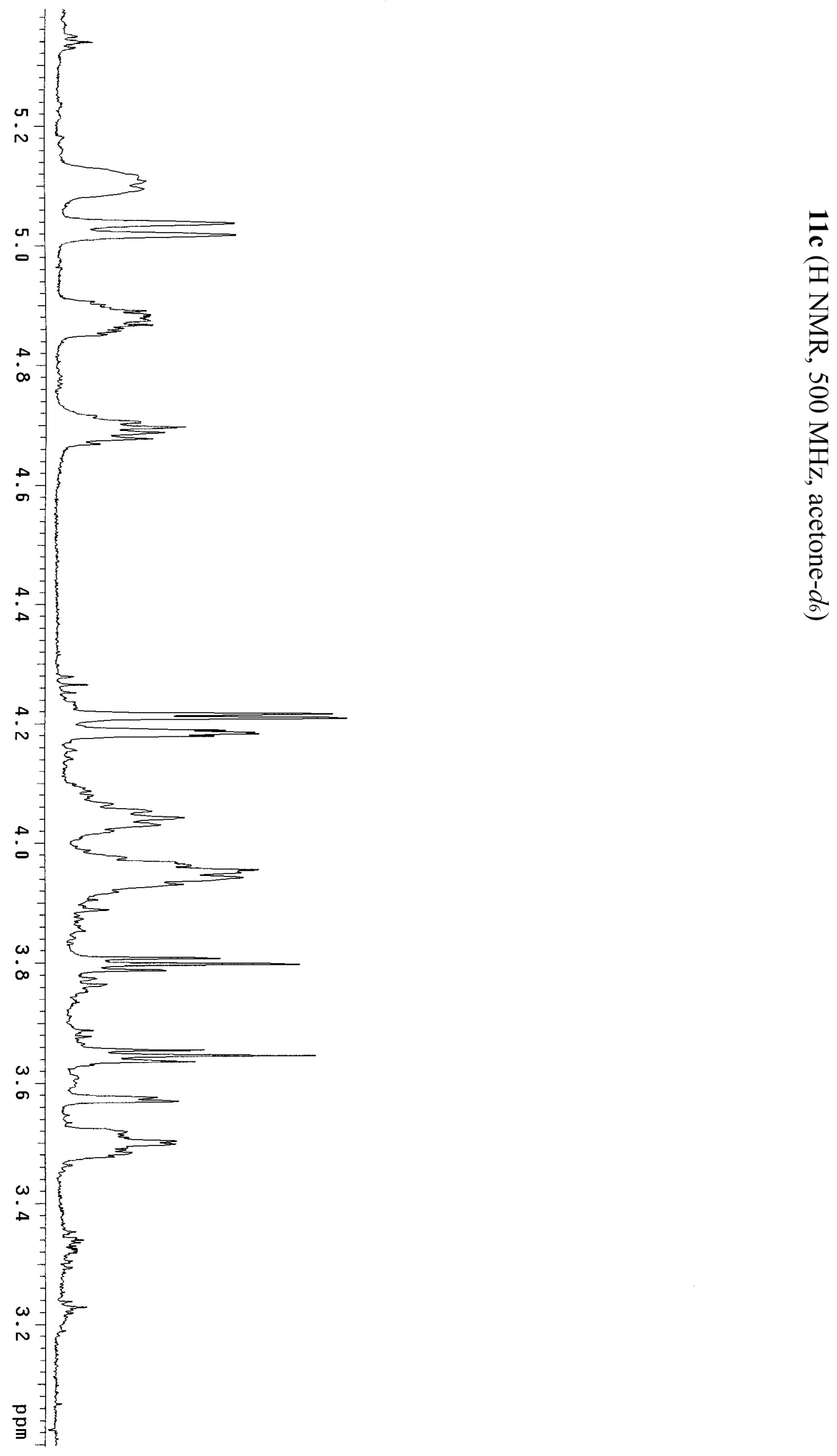


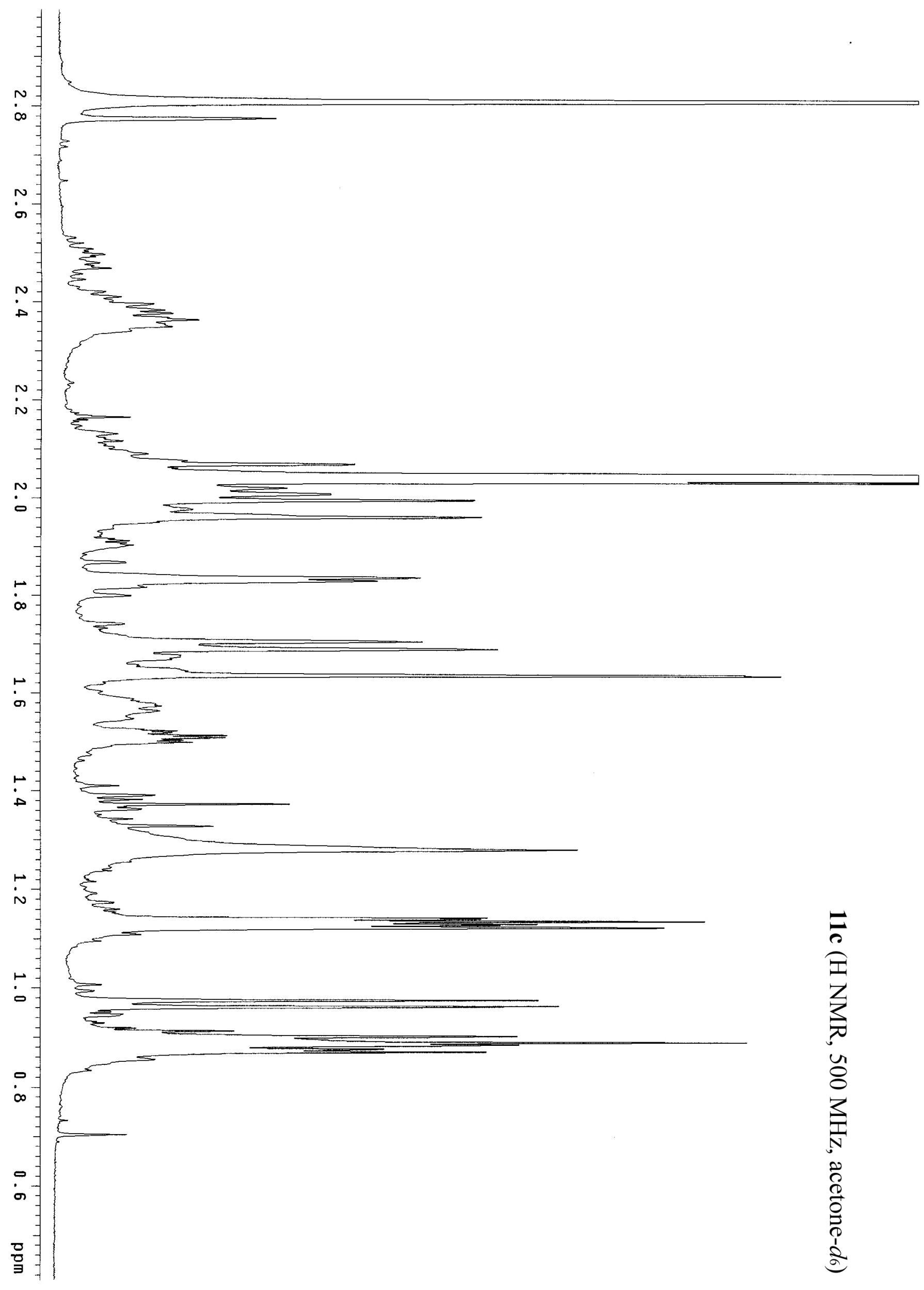




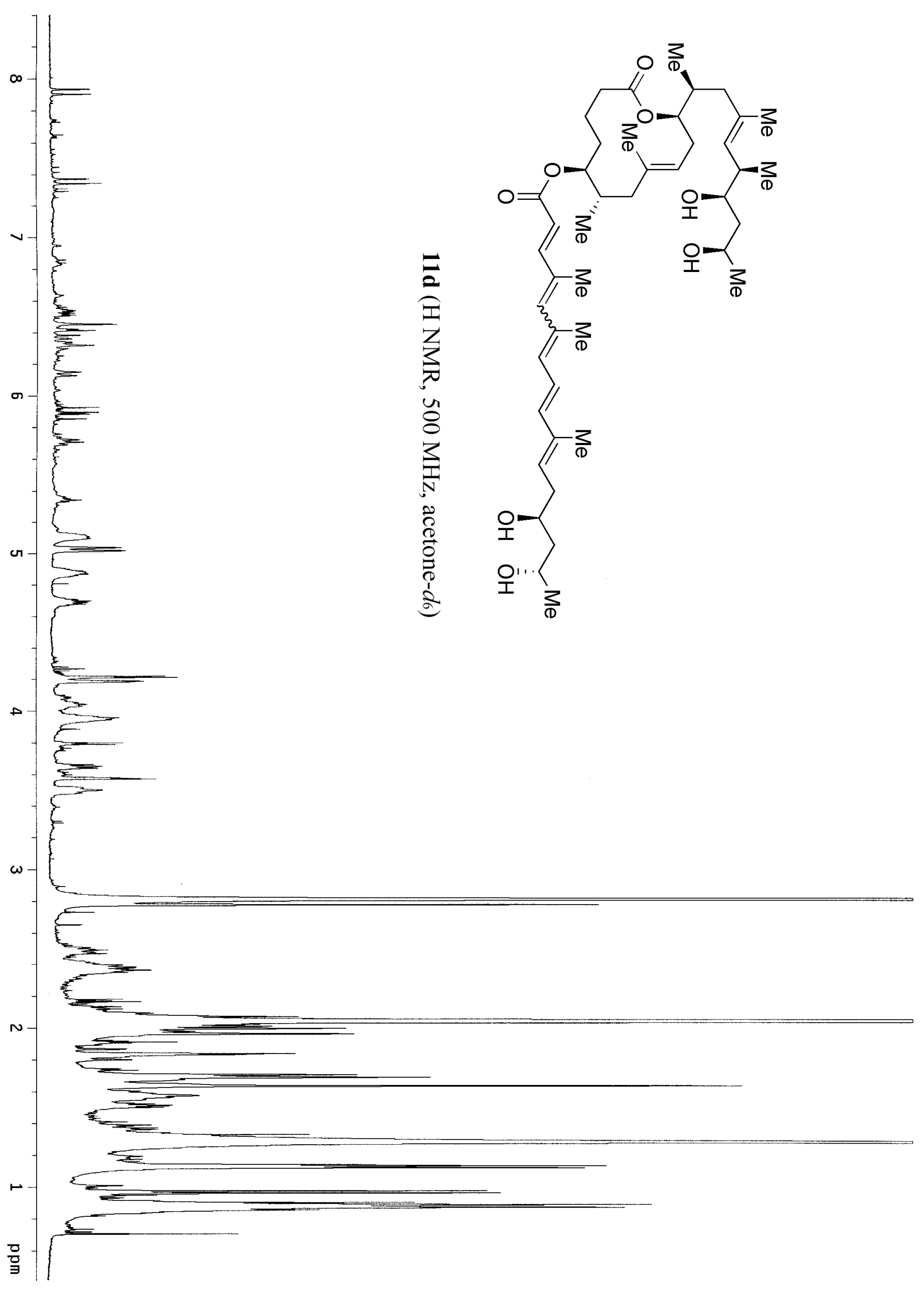



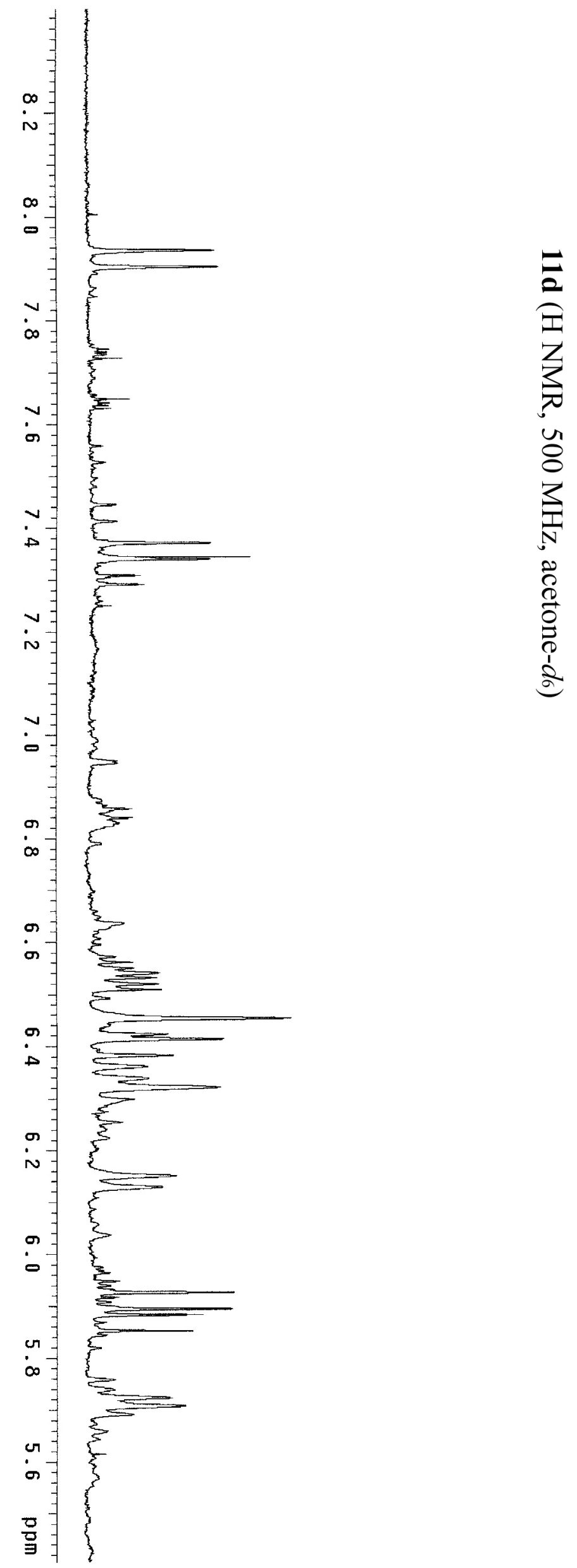


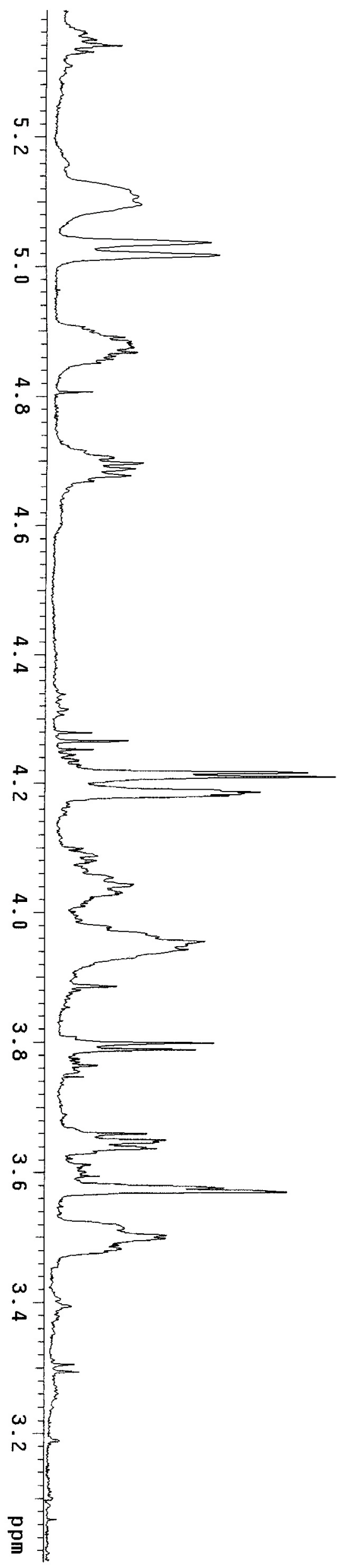

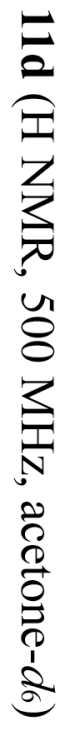




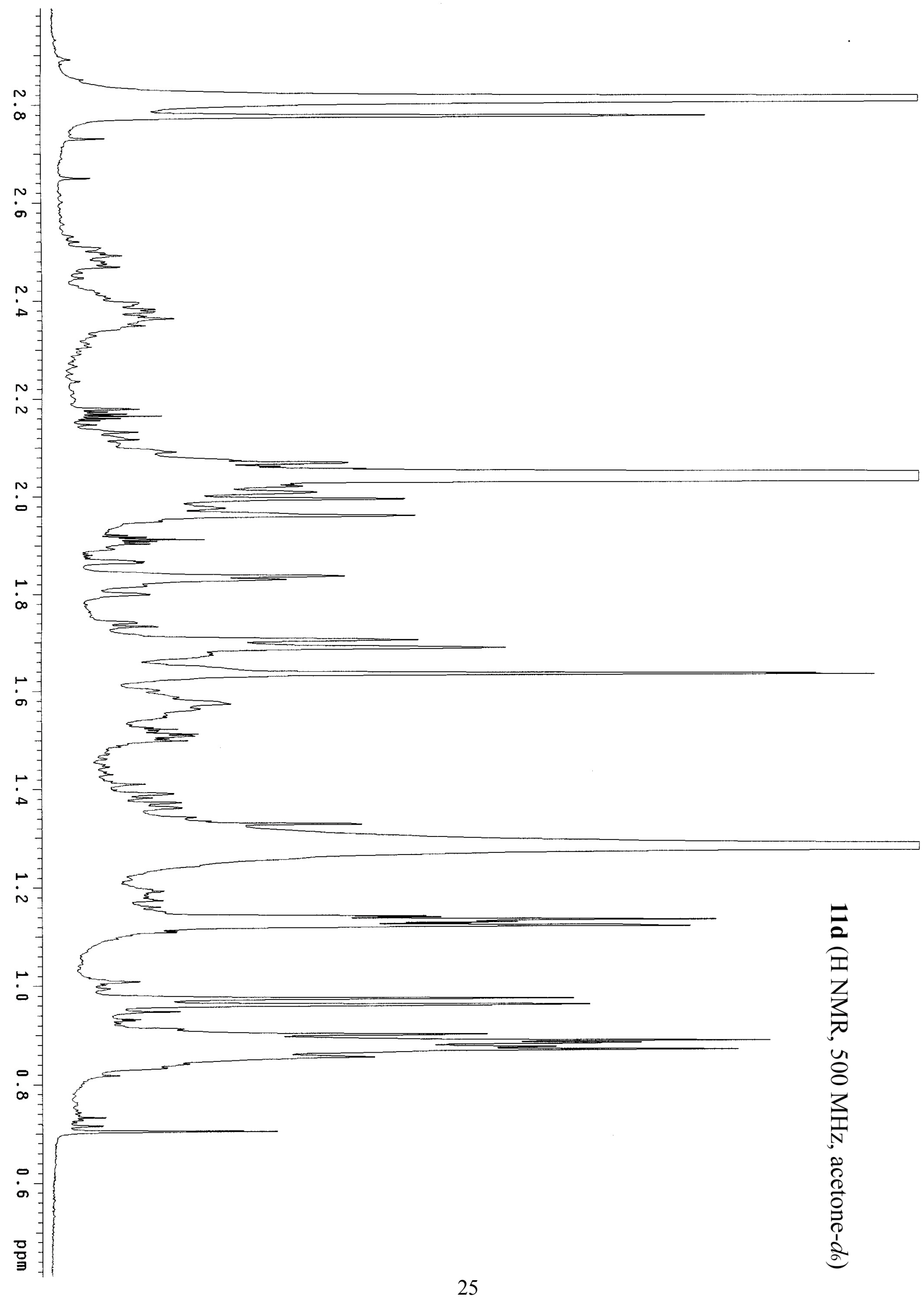




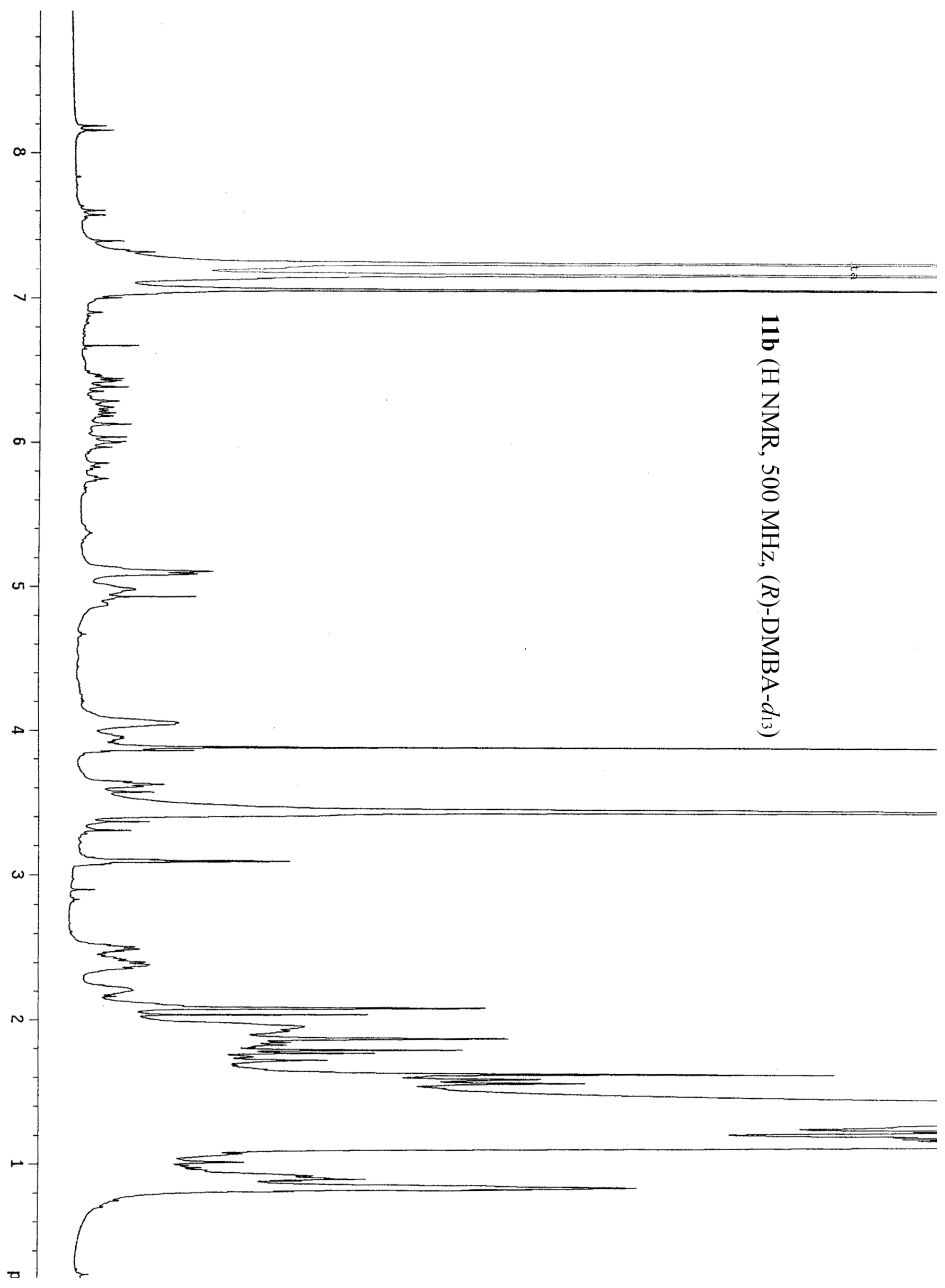




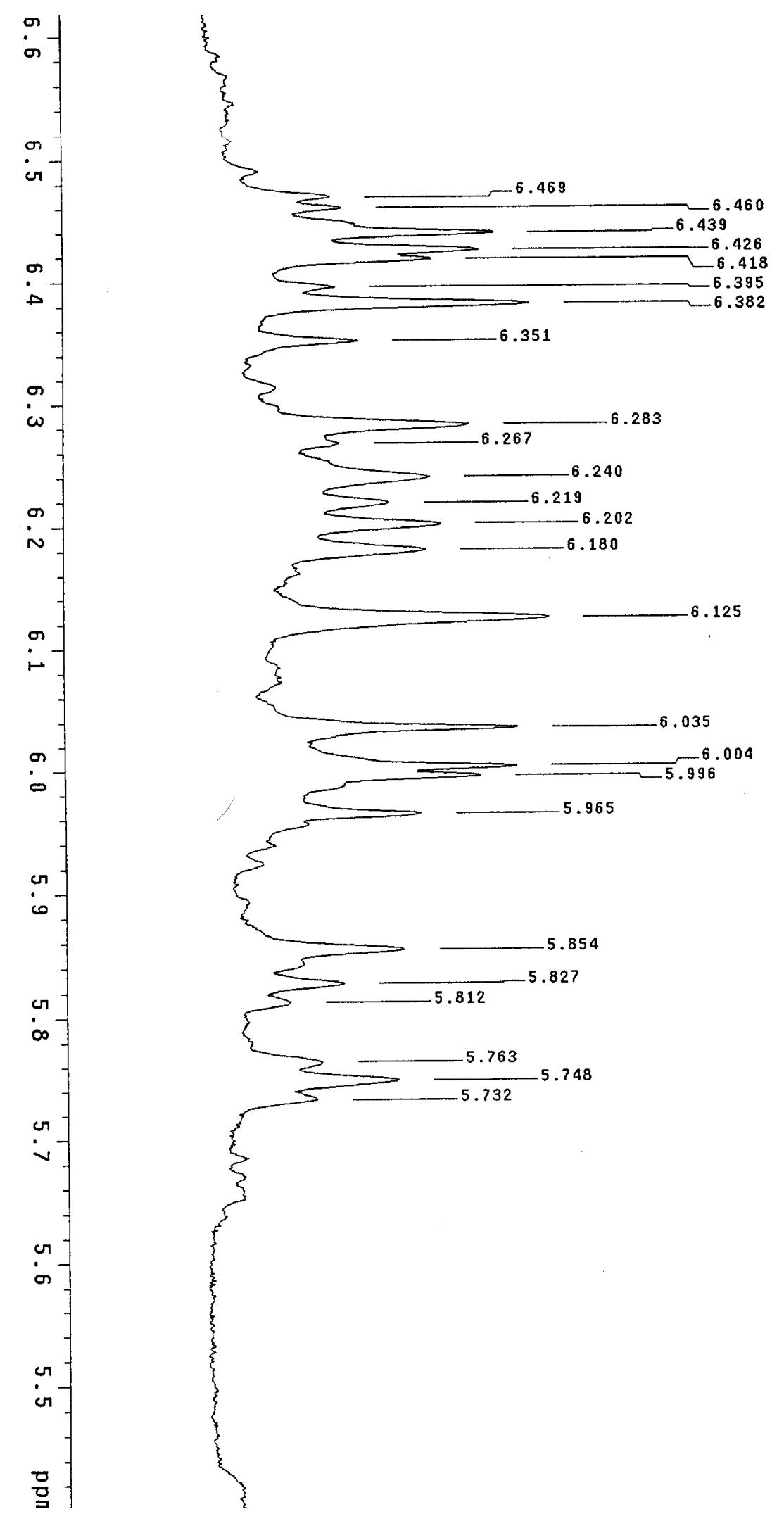

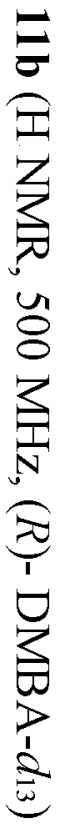




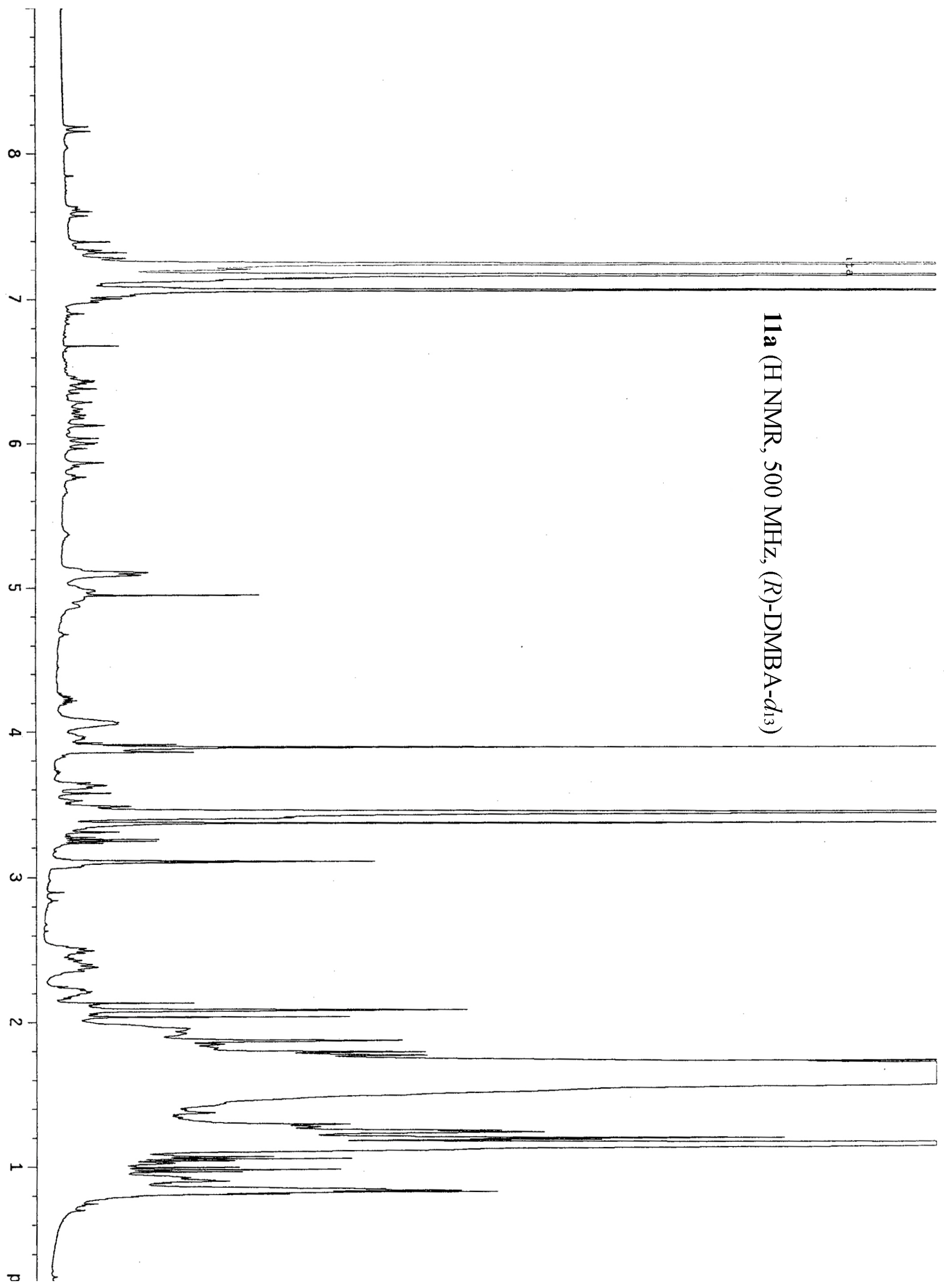




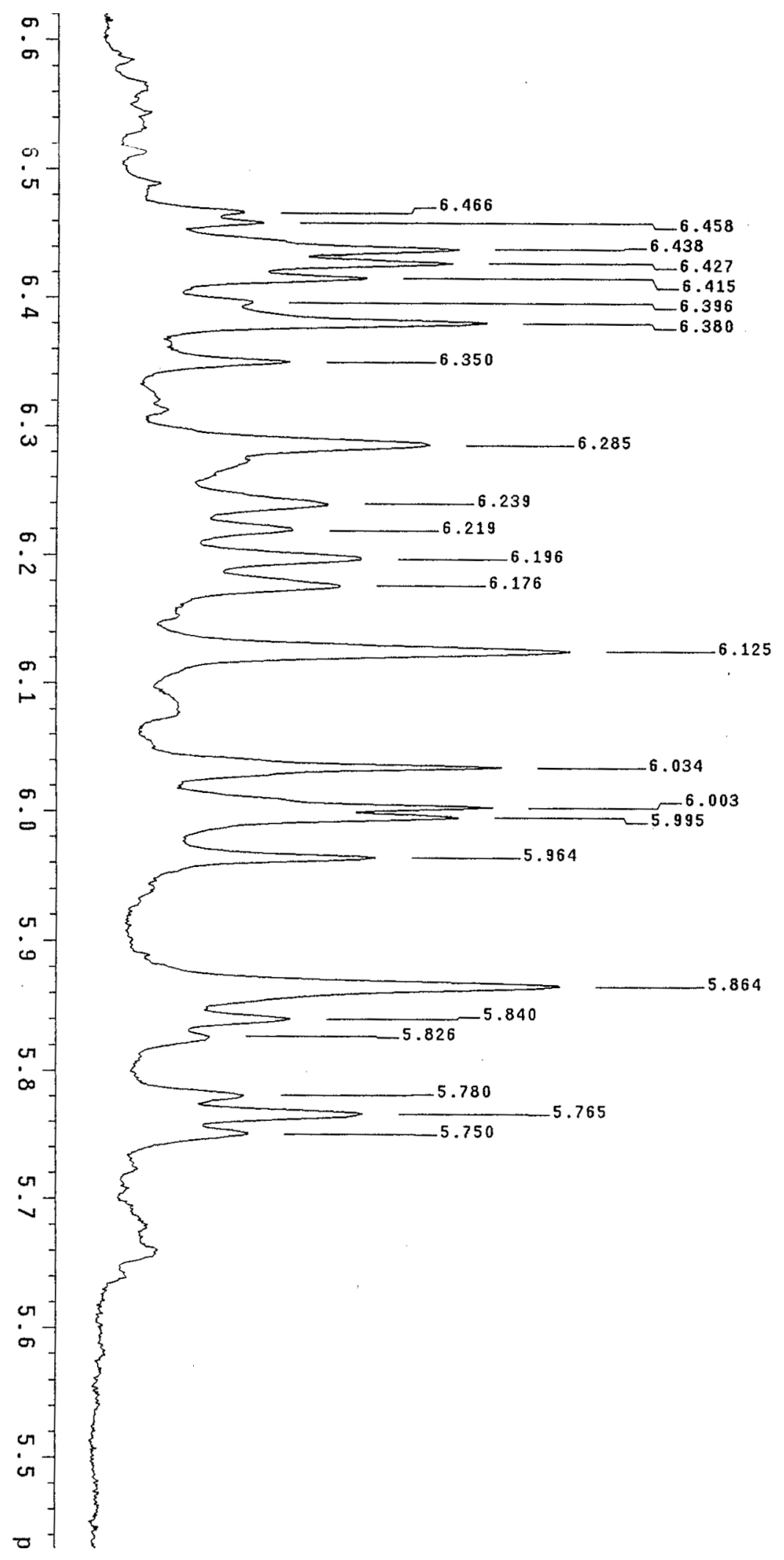

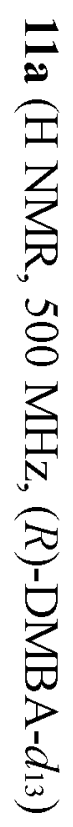




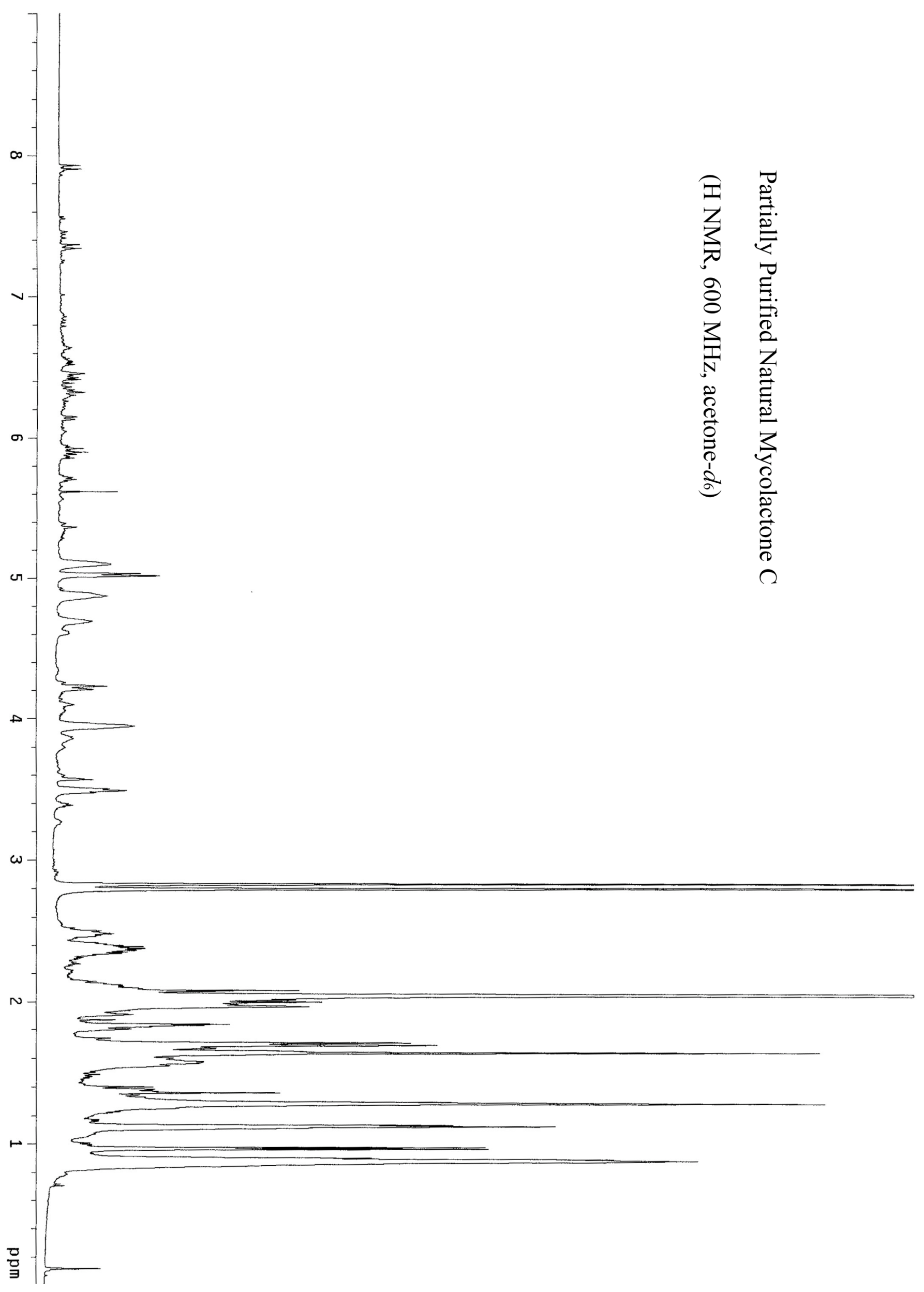




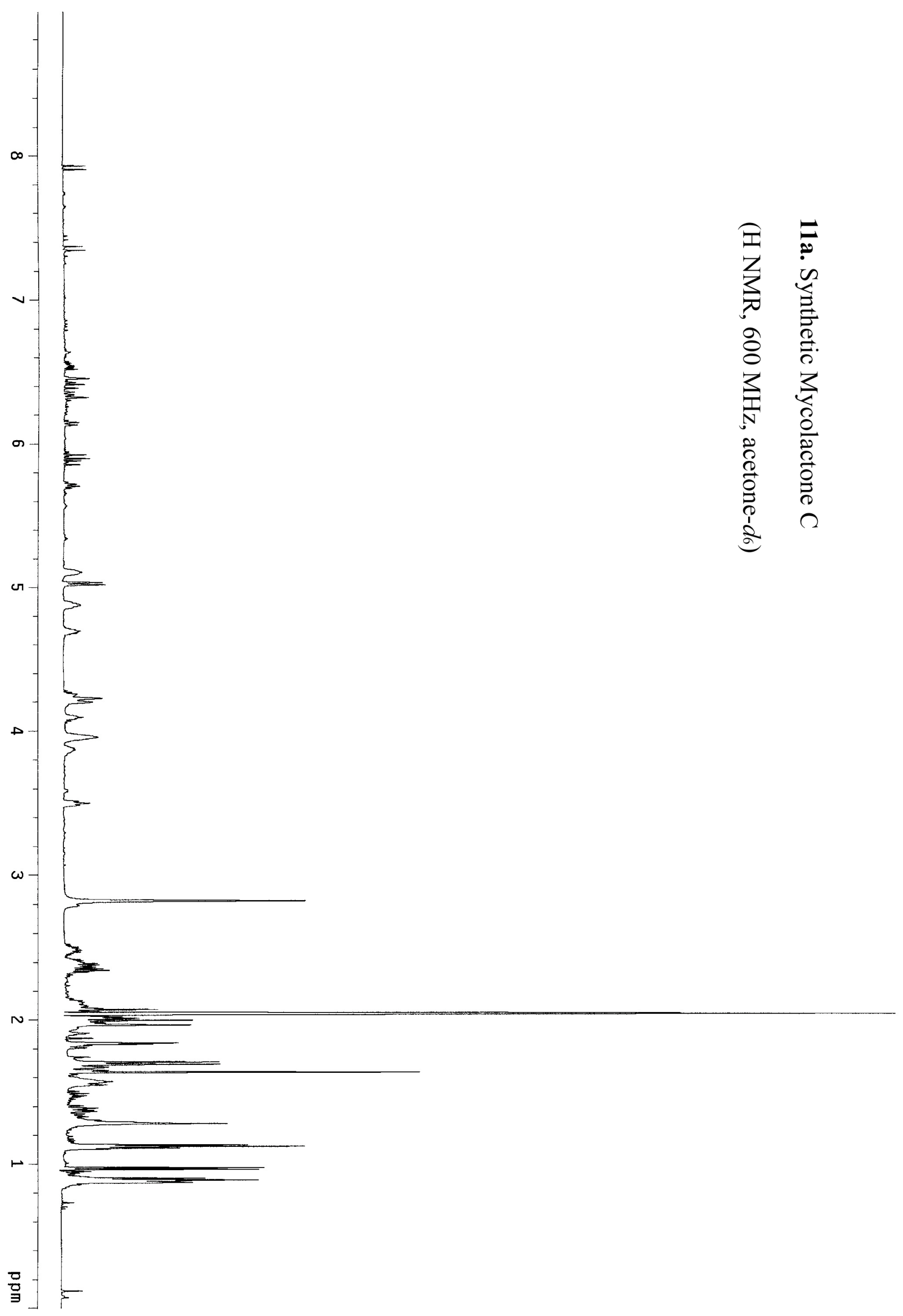

